\title{
Predicting modular functions and neural coding of behavior from a synaptic wiring diagram
}

Ashwin Vishwanathan ${ }^{1, \dagger}$, Alexandro D. Ramirez $z^{2,{ }^{*}}$, Jingpeng $\mathrm{Wu}^{1,{ }^{*}, \S}$, Alex Sood ${ }^{3}$, Runzhe Yang ${ }^{1,4}$, Nico Kemnitz $^{1}$, Dodam $\mathrm{Ih}^{1}$, Nicholas Turner ${ }^{1,4}$, Kisuk Lee ${ }^{1,5}$, Ignacio Tartavull ${ }^{1}$, William M. Silversmith ${ }^{1}$, Chris S. Jordan ${ }^{1}$ Celia David ${ }^{1}$, Doug Bland ${ }^{1}$, Mark S. Goldman ${ }^{3, \ddagger}$, Emre R. F. Aksay ${ }^{2,+,}$, H. Sebastian Seung ${ }^{1,4, \ddagger}$, the Eyewirers.

1. Princeton Neuroscience Institute, Princeton University, Princeton, NJ, USA. ; 2. Institute for Computational Biomedicine and the Department of Physiology and Biophysics, Weill Cornell Medical College, New York, New York, USA.; .3. Center for Neuroscience, University of California at Davis, Davis, California, USA.; 4. Computer Science Department, Princeton University, Princeton, NJ, USA.; 5. Brain \& Cognitive Sciences Department, Massachusetts Institute of Technology, Cambridge, MA, USA.

†Correspondence to vishwanathan.ashwin@gmail.com, msgoldman@ucdavis.edu and ema2004@med.cornell.edu

*These authors contributed equally

¥Senior authors

§Present address: Center for Computational Neuroscience, Flatiron Institute, NY, USA

\begin{abstract}
How much can connectomes with synaptic resolution help us understand brain function? An optimistic view is that a connectome is a major determinant of brain function and a key substrate for simulating a brain. Here we investigate the explanatory power of connectomics using a wiring diagram reconstructed from a larval zebrafish brainstem. We identify modules of strongly connected neurons that turn out to be specialized for different behavioral functions, the control of eye and body movements. We then build a neural network model using a synaptic weight matrix based on the reconstructed wiring diagram. This leads to predictions that statistically match the neural coding of eye position as observed by calcium imaging. Our work shows the promise of connectome-based brain modeling to yield experimentally testable predictions of neural activity and behavior, as well as mechanistic explanations of low-dimensional neural dynamics, a widely observed phenomenon in nervous systems.
\end{abstract}

\section{Introduction}

It has become a truism that the connectome is "necessary but not sufficient" for understanding the brain (Bargmann 2012; Briggman and Bock, 2012). This innocuous assertion includes a range of divergent views. Some declare that the connectome is "completely insufficient" (Nassim, 2018) and only a weak constraint at best. Others argue that the connectome is a major determinant of brain function (Denk et al., 2012), and the proper starting point for brain simulation (Seung, 2012). Due to technological advances in connectomics (Lee et al., 2019; Xu et al., 2020), the debate is moving into the domain of empirical study. Here we investigate the power of connectome-based modeling of brain function using a wiring diagram reconstructed from a larval zebrafish brainstem.

We reconstructed the synaptic connectivity of 3000 neurons from a 3D electron microscopic image. Despite highly intermingled cell bodies and arbors, the high resolution of the data enables graph clustering algorithms to identify two modules with stronger connectivity within than between modules. The modules are biologically validated using information about known cell types, and turn out to be specialized for different behaviors: one for eye movements and the other for body movements. The oculomotor module is in turn subdivided into two 
submodules, which appear to be specialized for movements of the two eyes. Our linking of structure and behavior by modularity analysis at synaptic resolution is novel in the vertebrate nervous system. It is especially remarkable in the brainstem reticular formation, which was regarded by classical anatomists as "undifferentiated" (Allen, 1932) or "diffuse" (Ramón-Moliner and Nauta, 1966). Previous findings of functionally specialized modules by connectivity analysis at synaptic resolution were confined to invertebrate nervous systems (Varshney et al., 2011. Jarrell et al., 2012; Pavlovic et al., 2014).

Having found modules, we then consider whether the wiring diagram can elucidate more detailed aspects of oculomotor function. When relating the wiring diagram to function, a common approach starts by extracting rules of synaptic connectivity between neuronal cell types, and then applies these rules to explain function (Seung, 2009). This approach has worked well for direction selectivity in the retina (Briggman et al., 2011; Kim et al. 2014; Ding et al., 2016), but is not guaranteed to generalize to other vertebrate brain structures that seem less stereotyped and precisely organized than the retina. Here we bypass connectivity rules and take a more direct approach: use the wiring diagram to estimate a synaptic weight matrix characterizing physiological interactions between neurons, and literally insert that matrix into a network model incorporating a minimal number of additional constraints from physiology.

Although the approach is naive, we use it to create a network model that predicts how eye position is encoded in neural activity. There are numerous reasons why our approach might have failed. We estimate the physiological strength of a connection based on the number of synapses involved, which is a rather crude measure. The model largely neglects the complexities of nonlinear dendritic integration, neuromodulation, and many other aspects of biophysics and biochemistry of neurons (Bargmann and Marder, 2013). Surprisingly, the predictions turn out to be statistically consistent at a population level with neural activity recorded by calcium imaging of larval zebrafish during oculomotor behavior. As far as we know, this is the first time that a vertebrate wiring diagram has been used to create a neural network model that predicts the encoding of behavior in neural activity. This success shows the promise of connectome-based brain modeling, an approach that should become even more powerful as more biophysical realism is incorporated.

The oculomotor system is of broad conceptual interest because it is a classic example of low dimensional neural activity dynamics (Seung, 1996). Low dimensional dynamical attractors have been proposed to underlie a large range of computations, from storage of working memory to reduction of noise in sensory and cognitive representations (Yoon et al., 2013; Daie et al., 2015; Kim et al., 2017; Green et al., 2017). However, it remains mysterious how these attractors are created at the level of individual cells and circuits. Furthermore, little attention has been paid to what features of the circuitry lead to the observed low-dimensional patterns of activity in recurrently connected networks and their downstream targets. Our reconstructed wiring diagram allows us to address these questions and thereby provides unprecedented insights into the cellular resolution circuitry governing the generation and transmission to target structures of low-dimensional attractors hypothesized to underlie a broad range of cognitive and non-cognitive tasks.

\section{Results}

\section{Neuronal wiring diagram reconstructed from a vertebrate brainstem}

We applied serial section electron microscopy (EM) to reconstruct synaptic connections between neurons in a larval zebrafish brainstem (Figure 1). The imaged dataset targeted a volume that is known to include neurons involved in eye movements (Schoonheim et al., 2010; Miri et al., 2011a; Daie et al., 2015; Lee et al., 2015; Vishwanathan et al., 2017). First, the dataset of Ref. (Vishwanathan et al., 2017) was extended by additional imaging of the same serial sections. By improving the alignment of EM images relative to our original work, we created a 3D image stack that was amenable to semiautomated reconstruction (Lee et al., 2019) of 100× more neurons than before. We trained a convolutional neural network to detect neuronal boundaries (Methods) (Lee 
et al., 2019), and used the output to generate an automated segmentation of the volume. To correct the errors in the segmentation, we repurposed Eyewire, which was originally developed for proofreading mouse retinal neurons (Kim et al., 2014). Eyewirers proofread 3000 objects, which included neurons with cell bodies in the volume as well as "orphan" neurites with no cell body in the volume (Figure 1A). We will refer to all such objects as "nodes" of the reconstructed network. Convolutional networks were used to automatically detect synaptic clefts, and assign presynaptic and postsynaptic partner nodes to each cleft (Turner et al., 2020) (Figure 1B-D, Methods). The reconstructed dataset contained 2824 nodes, 44949 connections between pairs of nodes, and 75163 synapses. Most connections (65\%) involved just one synapse, but some involved two (19\%), three $(7.9 \%)$, four $(3.7 \%)$, or more $(4.0 \%)$ synapses, up to a maximum of 21 (Figure S1A).

After registration of our EM volume to the Z-Brain reference atlas (Randlett et al., 2015) (Figure S2), several important groups of neurons and neurites were identified (Methods). It was straightforward to recognize two populations of reticulospinal (RS) projection neurons involved in escape behaviors and controlling movements of the body axis. The neurons of one population were large and dorsally located, and of the other were smaller and ventromedially located (Figure $1 \mathrm{H}$ ) Comparison with transgenic lines and cranial nerves in the atlas yielded the abducens (ABD) neurons controlling extraocular muscles, and Descending Octaval (DO) neurons that mediate optokinetic and vestibular signals (Pastor et al., 2019) as part of the sensorimotor transformations needed to control eye movement (Figure 1E-H; Figure S3, S4; Methods).

\section{Axial and oculomotor modules in the brainstem}

Before looking for modularity, we divided the reconstructed neurons into "center" and "periphery" (Methods). Neurons in the "center" (540 neurons) are recurrently connected to other reconstructed neurons that are expected to predominate in establishing collective dynamics. Neurons in the "periphery" (2344 nodes), in contrast, are involved in feedforward pathways that supply input to the center, transmit output from the center, or have negligible recurrent connectivity as quantified by eigenvector centrality (62 nodes). For example, the periphery includes $A B D$ neurons, which mediate a downstream pathway to the extraocular muscles, as mentioned above. The periphery also includes most reticulospinal projecting neurons, such as the Mauthner cell, MiV1 and MiV2 cells (Figure $1 \mathrm{H}$ ).

Complex systems, both biological (Wagner et al., 2007) and artificial (Baldwin et al., 2000), can often be divided into modules, such that interactions within modules are stronger than interactions between them. While such modules are structurally defined, they often turn out to be functionally specialized. To identify modules in our reconstructed wiring diagram, we applied a graph clustering algorithm to the center of the network (Methods). This analysis revealed a block structure in the connection matrix (Figure 2A). The diagonal blocks of the matrix contain the connections within modules, and the off-diagonal blocks contain connections between modules. The diagonal blocks are more densely connected than the off-diagonal blocks, meaning that within-module connectivity is stronger than between-module connectivity, which is what the graph clustering algorithm is meant to achieve. We designated the two modules as modA and modO, for reasons explained below.

For biological validation of the modules, we checked them against information that was not used by the clustering algorithm. Namely, we checked the relation of the modules to two neuron classes (RS and ABD) identified above (Figure 2A). RS neurons in the periphery received much stronger connectivity from modA than from modO (Figure 2A, RS; Figure S8). Of the 10 smaller RS neurons contained in the center, all were in $\operatorname{modA}$, and none were in modO (Figure S8A, black arrows). Furthermore, the $15 \mathrm{RS}$ neurons (small and large) contained in the periphery received much stronger connectivity from modA than from modO (Figure S8). Since the RS neurons are known to be involved in turning or swimming movements (Gahtan et al., 2002; Orger et al. 2008; Huang et al., 2013; Bhattacharyya et al., 2017), we propose that modA plays a role in movements of the body axis, and refer to it as the "axial module."

All 54 ABD neurons were in the periphery, and received much stronger connectivity from modO than from modA (Figure 2A). All 34 of the DO neurons (Figure 2c) were members of modO; none were in modA. ABD neurons drive extraocular muscles either directly or through a disynaptic pathway. DO neurons are secondary 


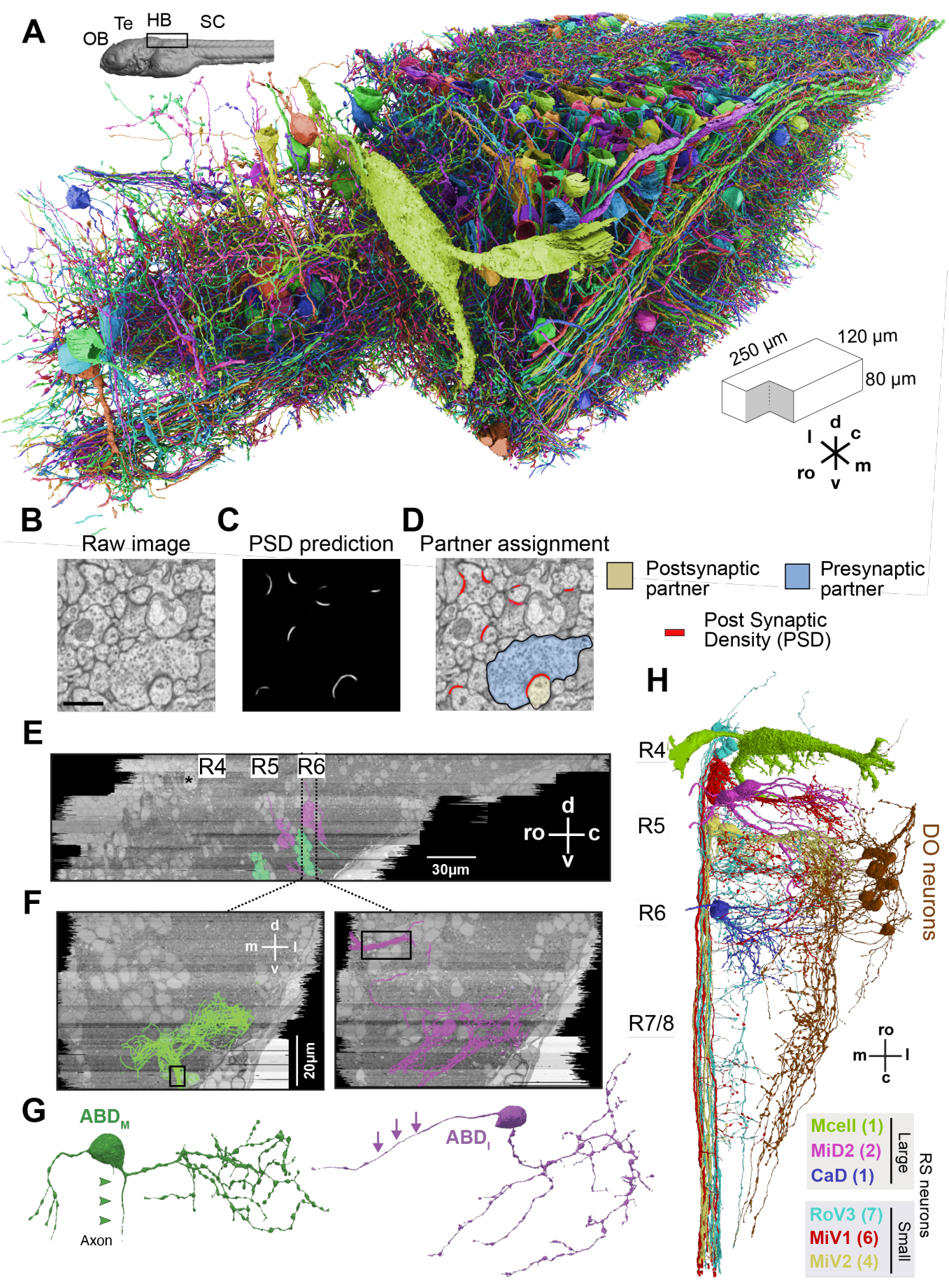

Figure 1: EM reconstructions of brainstem neurons. A. 3D rendering of reconstructed neurons. Large green cell body in the foreground is the Mauthner neuron (ro - rostral; c - caudal; d - dorsal; v - ventral; I - lateral; m - medial). Inset (top left) shows location of the unilateral EM volume (black box) relative to the olfactory bulb (OB), tectum (TE), hindbrain (HB), and spinal cord (SC). B-D. Automatic synapse detection and partner assignment. B) Raw EM image. Scale bar is 750nm. C) Postsynaptic densities (PSDs) identified by a convolutional net. D) PSDs (red) overlaid onto the original raw image, together with an exemplar presynaptic (blue) and postsynaptic (yellow) partnership identified by a second convolutional net. E. Sagittal view of identified abducens motor ( $A B D_{M}$, green) and abducens internuclear $\left(A B D_{l}\right.$, magenta) neurons overlaid over representative EM planes ( $R$ - rhombomere; ${ }^{*}$ - Mauthner cell soma). F. Coronal planes showing the locations of the $\mathrm{ABD}_{\mathrm{M}}$ (left) and $A B D_{\text {I }}$ (right) neurons at the planes indicated by dotted black lines in e sagittal view. Black boxes highlight nerve bundles from these populations. G. Representative $A B D_{M}$ and $A B D_{1}$ neurons with arrows indicating the axons. H. Reconstructions of large and small reticulospinal (RS) neurons and dorsal octaval (DO) neurons. 


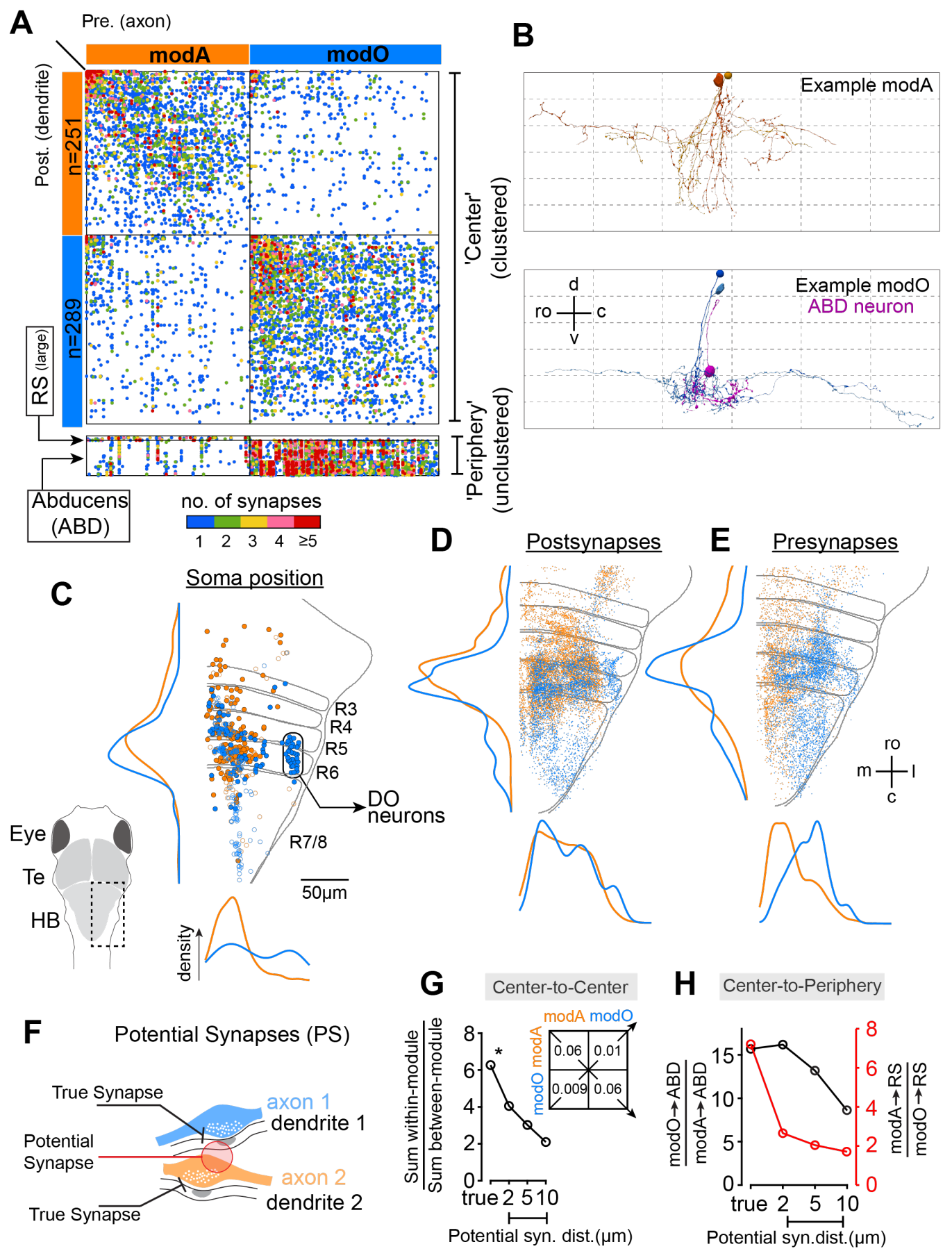

Figure 2: Modularity and functional specialization of interneurons. A. (Top) Matrix of connections in the 'center' of the wiring diagram, with neurons clustered into two modules (modA, modO). (Bottom) Matrix of connections from center to select reticulospinal (RS) and abducens (ABD) neurons in the periphery. B. (Top) Example connected pairs of modA neurons. (Bottom) Example connected pair of modO neurons (light and dark blue) and the overlap of their axons with the dendrite of an abducens internuclear cell (magenta) (ro - rostral; c - causal; $d$ - dorsal; $v$ - ventral). Grid in the background is the same in both panels to facilitate comparison. C. Locations of reconstructed neuron soma (modA - orange, modO - blue) projected onto the horizontal plane and 1D densities along the mediolateral (bottom) and rostrocaudal (right) axes (DO - descending octaval; R - rhombomere). Closed circles are neurons with complete somas inside the reconstructed EM volume. Open circles are locations of the primary neurites exiting the top of the EM volume for cells with somas above the volume. Inset cartoon shows the region of the hindbrain in the figure. Te - tectum, HB - hindbrain. D. Postsynapses of neurons in modA and modO along with $1 \mathrm{D}$ densities. Every $5^{\text {th }}$ postsynaptic density is plotted for clarity. E. Presynapses of neurons in modA and modO along with $1 \mathrm{D}$ densities. Every $10^{\text {th }}$ presynaptic terminal is plotted for clarity. ( $\mathrm{m}$ - medial; I - lateral). F. Schematic illustrating the definition of a potential (i.e. false) synaptic connection identified when a presynaptic terminal (e.g. axon 2) is proximal (red) to a postsynaptic density (e.g. dendrite 1) but not actually in contact with it. G. Ratio of the number of within-module to the number of between-module synapses versus threshold distance for true and potential synapses. Table lists the actual true synapse (TS) numbers for data point with asterisk *. H. Ratio of numbers of synapses from neurons in modA and modO to peripheral neurons (ABD, RS). 
vestibular neurons, which provide input to the vestibulo-ocular reflex. We therefore propose that modO plays a role in eye movements, and will refer to it as the "oculomotor module."

The modules in Figure 2 were obtained with the Louvain algorithm for graph clustering (Blondel et al., 2008; Reichardt and Bornholdt, 2006; Rubinov and Sporns, 2010). Similar modules are obtained when spectral clustering (Chung, 2005) or a degree-corrected stochastic block model (Peixoto, 2014) are used (Figure S7). As we will see later, the binary division into two modules is the first step in a hierarchical clustering procedure. (For comparison with flat clusterings, see Figure S5.)

It does not appear that modA and modO can be regarded as traditional brain "nuclei," because their somas are often intermingled and are highly distributed, extending rostrocaudally over all rhombomeres in the EM volume (Figure 2C). Postsynapses and presynapses are even more diffusely distributed than somas (Figure 2D, E). However, presynapses of $\bmod A$ and modO do exhibit some spatial segregation along the mediolateral axis (Figure 2E), which reflects an underlying spatial organization of axonal arbors (data not shown). Therefore we decided to probe to what extent spatial organization could be contributing to modularity.

To quantify modularity, we defined an index of wiring specificity as the ratio of the sum of within-module synapse densities to the sum of between-module synapse densities. Here synapse density is defined as the number of synapses normalized by the product of presynaptic and postsynaptic neuron numbers. The wiring specificity index was roughly 6 for the center neurons (Figure $2 \mathrm{G}$ ), based on actual synapses. We defined a "potential synapse" as a presynapse and a postsynapse within some threshold distance of each other (Figure $2 F$ ), similar to the conventional definition as an axo-dendritic apposition within some threshold distance (Stepanyants and Chklovskii, 2005). Then we computed the wiring specificity index based on potential synapses rather than actual synapses. The index dropped to less than 3 for potential synapses defined by a distance threshold of $5 \mu \mathrm{m}$, and close to 2 at a distance threshold of $10 \mu \mathrm{m}$ (Figure $2 \mathrm{G}$, center-to-center).

The implication is that the division into modA and modO can be explained by spatial organization, as long as location information is precise to within a few microns or less (Motta et al., 2019). On the other hand, the coarse (tens of microns) mediolateral segregation of presynapses evident in Figure 2E must contribute little to modularity.

We similarly defined an index of wiring specificity for peripheral populations as the synapse density from preferred partner in the center divided by the synapse density from non-preferred partner in the center. This wiring specificity index decreases greatly for peripheral RS neurons when potential synapses are considered, but the decrease is more modest for ABD neurons (Figure $2 \mathrm{H}$, center-to-periphery). We also found some statistical differences between modA and modO in total arbor length, synapse size, and synapse distance from soma (Figure S6A, B).

To further validate our claims regarding function, we performed calcium imaging throughout the hindbrain in a separate set of 20 age-matched animals. We resorted to comparison with other animals because our calcium imaging of the neurons in the EM volume (Vishwanathan et al., 2017) had highly incomplete coverage due to technical limitations. Recordings were obtained while the animals performed spontaneous eye movements in the dark, with activity ranging from neurons that exhibited bursts during saccades to those with perfectly persistent firing during fixations (Ramirez and Aksay, 2018). The images from 20 age-matched animals were combined by registering them to a reference atlas (Randlett et al., 2015). We extracted a map for eye movement signals (Methods) that was complete, in the sense that each hindbrain voxel was covered by at least three fish (Methods). Relative to modA somas, modO somas were more than twice as likely to be neighbors with somas in the map of eye movement signals (Figure S9). While this preference is not extremely strong, it seems reasonably strong given that there is intermingling of modA and modO somas (Figure $2 \mathrm{C}$ ), and registration to the reference atlas is unlikely to remove all soma location variability across individuals. The preference disappeared when soma locations were artificially jittered by more than 10um (Figure S9). 


\section{Submodules of the Oculomotor network}

There is a rich repertoire of oculomotor behaviors, which vary in speed of movement, patterns of binocular coordination, and other properties. Motivated by this functional diversity, we applied the same graph clustering algorithm employed in Figure 2A to modO. Doing this revealed the presence of two submodules characterized by strong within-submodule connectivity and weak between-submodule connectivity (Figure $3 \mathrm{~A}$ ).

For biological validation of the submodules, we examined the connectivity from modO to ABD neurons. This center-periphery connectivity provides independent validation, because the graph clustering algorithm relied only on intra-center connectivity. The abducens complex is composed of two groups, the motor neurons $\left(A B D_{M}\right)$ that directly drive the lateral rectus muscle of the ipsilateral eye, and the abducens internuclear neurons $\left(A B D_{1}\right)$ that indirectly drive the medial rectus muscle of the contralateral eye through a disynaptic pathway. Increased activities in both $A B D_{M}$ and $A B D_{\text {| }}$ neurons drive eye movements toward the side of the brain on which the neurons reside ('ipsiversive' movements). Neurons in one submodule of modO preferentially connected to $A_{B D}$ neurons, while neurons in the other submodule preferentially connected to $A B D_{1}$ neurons. This is evident from both visual inspection (Figure $3 \mathrm{~A}$ ) and quantitative analysis (Figs. $3 \mathrm{~A}, \mathrm{~F}$ ). We therefore refer to the submodules as motor-targeting $\left(\operatorname{modO}_{M}\right)$ and internuclear-targeting $\left.(\operatorname{modO})_{\mathrm{I}}\right)$, and suggest they are largely involved in controlling movements of the ipsilateral and contralateral eye, respectively.

The spatial layout of somas, postsynapses and presynapses for these submodules is shown in Figure $3 \mathrm{~B}$ D. We again quantified the contribution of spatial organization to wiring specificity using the potential synapse formalism, and found that the division of $\operatorname{modO}$ into $\operatorname{modO}_{M}$ and $\operatorname{modO}_{\text {I }}$ requires spatial precision of a few microns (Figure 3 E, center-to-center). A similar finding holds for wiring specificity from modO to ABD (Figure 3F, centerto-periphery). Preferences of individual neurons can be extreme: many modO cells connect to $A_{B D}$ only or $A B D_{1}$ only (Figure $3 \mathrm{G}$ ). There were some statistical differences between $\operatorname{modO}_{M}$ and $\operatorname{modO}_{1}$ in synapse size and synapse distance from the soma (Figure S6D, E).

\section{Predicting neural coding of eye position from the wiring diagram}

We hypothesized that modO contains the "neural integrator" for horizontal eye movements that transforms angular velocity inputs into angular position outputs. The necessity of such a velocity-to-position neural integrator (VPNI) was pointed out in the 1960s because sensory and command inputs to the oculomotor system encode eye velocity, whereas the extraocular motor neurons additionally carry an eye position signal (Robinson, 1968). The transformation of velocity into position is "integration" in the mathematical sense of Newtonian calculus. It has been suggested that this transformation is dependent on a relatively high degree of recurrent interactions within the VPNI. We note that the prevalence of recurrent interactions in modO was significantly higher than in modA (Figure 4A).

We developed a network model to assess if functional properties of VPNI neurons and their oculomotor partners could be predicted from connectomic information. The model consisted of four populations: DO neurons, VPNI cells, ABD interneurons, and ABD motoneurons (Figure 4B). DO neurons provide optokinetic and vestibular velocity signals to the abducens and VPNI; they exhibit little to no change in activity during spontaneous saccades and fixations (Pastor et al., 2019). VPNI neurons were identified as all neurons in modO excluding the DO cells; this was justified based on comparison of the extent of the reconstructed volume and functional maps of oculomotor cell types (see Methods). Abducens neurons receive input from both VPNI and DO neurons. We focused on predicting the relative strength of the eye position signal in these different populations during spontaneous fixation behavior.

A minimal set of physiological constraints were imposed on the model. The signs of the weights were chosen according to rationales described in the Methods. Briefly, all DO cells were inhibitory (Figure S3C, Methods) (Pastor et al., 2019), and the remaining cells were excitatory (Lee et al., 2015) (Figure S9C, Methods). The recurrent network model omitted interactions with neurons on the other side of the brain, based on previous physiological 
A

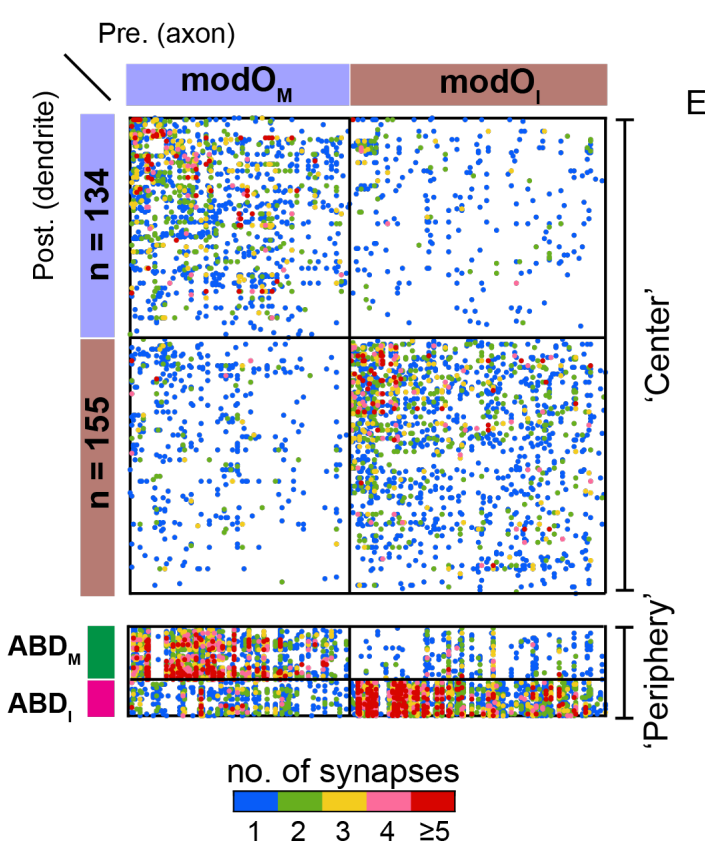

B

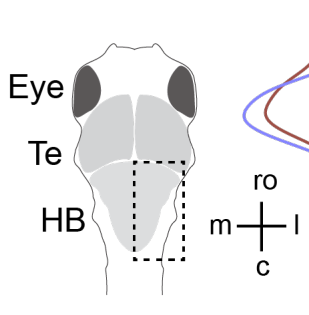

Soma position
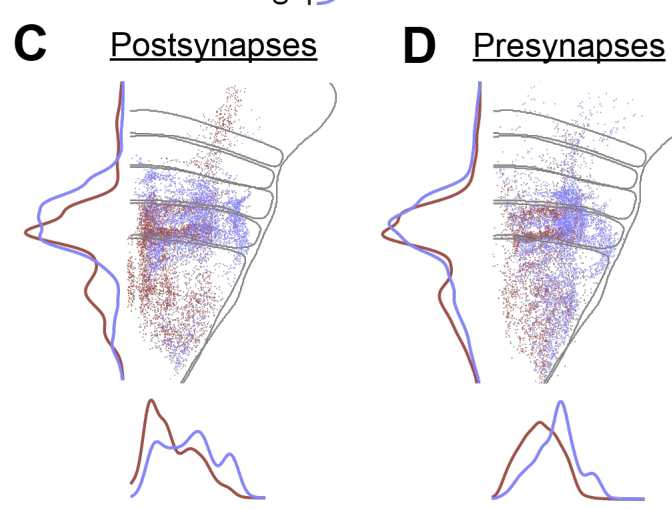
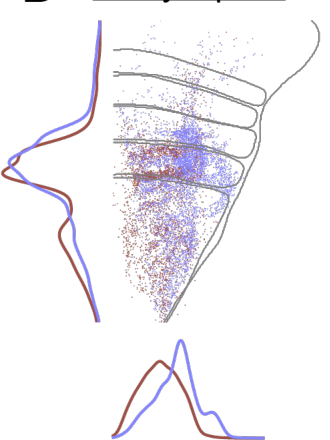
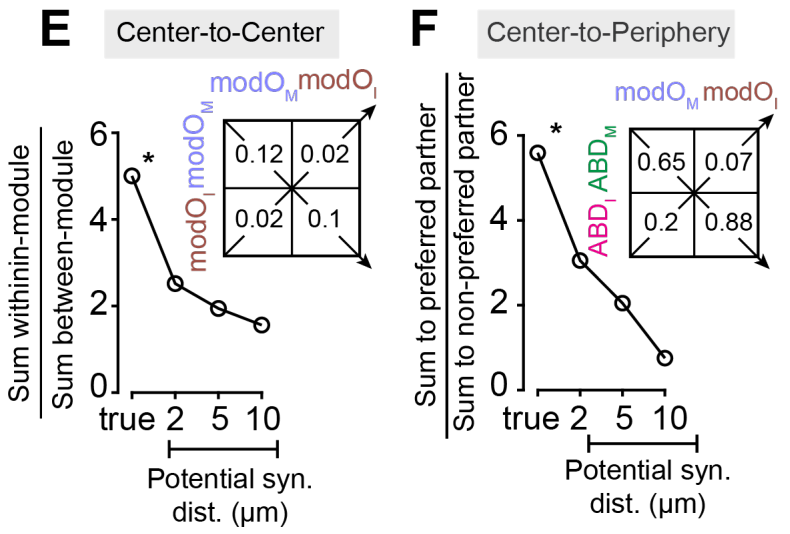

G Ocular Preference Index (OPI)

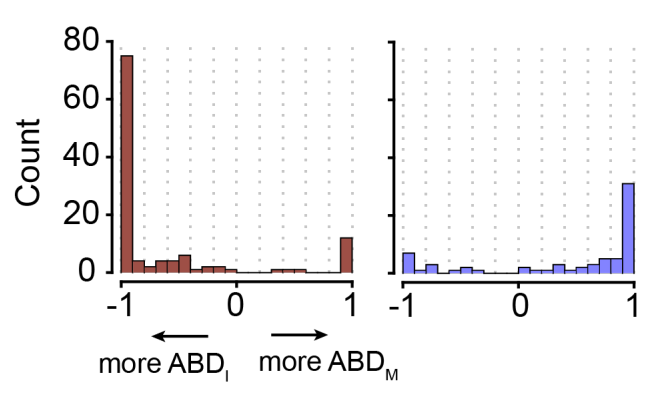

dist. $(\mu \mathrm{m})$

Figure 3: Submodules specialized for the two eyes. A. (Top) Matrix of connections within modO organized into two sub-modules termed $\operatorname{modO}_{M}$ and modO. (Bottom) Projections of $\operatorname{modO}_{M}$ and $\operatorname{modO}_{1}$ onto abducens motor neurons $\left(\mathrm{ABD}_{\mathrm{M}}\right)$ and abducens internuclear neurons $\left(A B D_{1}\right)$ populations. B. Locations of reconstructed neuron soma along with $1 \mathrm{D}$ densities for neurons within modO $\mathrm{M}_{\mathrm{M}}$ (blue) modO (brown). Symbols, inset, and orientation as in Figure 3C. C-D. Postsynaptic densities (C) and presynaptic terminals (D) in modO, and $\operatorname{modO}_{M}$. Every $5^{\text {th }}$ synaptic site was plotted for clarity. E. Ratio of the number of synapses within-modules $\operatorname{modO}_{M}$ and modO, to the number between-modules as a function of potential synapses distance. Table lists the actual true synapses numbers for data point with asterisk *. F. Ratio of the number of synaptic contacts between a modO submodule and its preferred peripheral partner vs those between a modO submodule and its non-preferred peripheral partner. Numbers in tables represent normalized synapse counts defined as the ratio of sum of all synapses in a block to the product of the number of elements in the block. G. Ocular Preference index (OPI) for modO $\mathrm{M}_{\mathrm{M}}$ and modO, neurons. DO vestibular neurons were not included. 
studies indicating that persistent activity in the oculomotor integrator can be generated independently by each half of the brain (Aksay et al., 2007a; Fisher et al., 2013; Gonçalves et al., 2014). Likewise, we omitted interactions between modO and modA, based upon their separate roles in controlling eye movement versus body movement behaviors (Orger et al., 2008; Huang et al., 2013; Pujala and Koyama, 2019) and the body-fixed nature of the oculomotor behavior being investigated.

To create a network model we first derived a synaptic weight matrix from our EM reconstruction as follows. Each element $W_{i j}$ of the weight matrix is the physiological strength of the connection received by neuron $i$ from neuron $j$. We approximated $W_{i j}$ as proportional to $N_{i j}$, the number of synapses received by $i$ from $j$. This approximation effectively assumes that all synapses involved in a connection have the same strength. Then, for each neuron $i$, we divided by $\Sigma_{k} N_{i k}$, where the sum includes all synapses received by neuron $i$, including those from neurons outside modO. Therefore the final synaptic weight matrix took the form $W_{i j}= \pm \beta \frac{N_{i j}}{\sum_{k} N_{i k}}$.

The normalizing factor in the denominator has two interpretations. Scaling the strength of an individual synapse by the total number of synapses incoming to a neuron is similar in concept to homeostatic synaptic scaling as observed experimentally (Turrigiano, 2012). Alternatively, the normalization can be viewed as a way of compensating for truncation of dendritic arbors by the borders of the EM volume. Due to the normalization, each recurrent connection strength $W_{i j}$ is proportional to the fraction of the total inputs that neuron $j$ provides to neuron $i$, and the sum $\Sigma_{j} W_{i j}$ over neurons in modO gives the "recurrent fraction" of input to neuron $i$ in $\operatorname{modO}$, defined as the fraction of synapses received from other modO neurons (Figure 4A. Average recurrent fraction within $\bmod A$ and $\operatorname{modO}$ are $\mu_{\bmod A}=0.06 \pm 0.05, \mu_{\operatorname{modO}}=0.11 \pm 0.08, p=1.2 \times 10^{-18}$, Wilcoxon-rank sum test).

Linear model neurons (Cannon et al., 1983) were used for simplicity because most VPNI cells have firing rates that vary linearly with eye position for ipsilateral eye positions (McFarland and Fuchs, 1992; Aksay et al. 2000). For each neuron, the slope of this linear rate-position relationship is known as the neuron's eye position sensitivity. At this point, we could have simulated the dynamics of the network to extract predictions of relative eye position sensitivities. This was unnecessary because the results of simulation can be derived analytically from an eigenanalysis of the synaptic weight matrix, following previous theoretical work (Seung, 1996). The eigenanalysis yielded predictions of the eye position sensitivities of DO, VPNI and downstream ABD neurons, up to an overall scale factor (Methods). Each of the four populations displayed a characteristic distribution of eye position sensitivities (Figure 4E).

To test the model prediction, we extracted eye position sensitivities from calcium imaging experiments conducted in other larval zebrafish (Methods). We assessed eye position sensitivity during spontaneous fixations in the dark (Figure 4C, D), when persistent firing depends strictly on the internal dynamics of the VPNI. Because the imaged neurons come from other individuals, they cannot be placed in one-to-one correspondence with neurons in our EM volume. Therefore we resorted to a population-level comparison. The distribution of eye position sensitivities for each population matched the model predictions quite well (Figure 4E). We only considered three classes of neurons (VPNI, DO, and $A B D$ ) in the experiments, which could not easily distinguish between $A B D_{M}$ and $A B D$, neurons. However, the bimodal distribution for the $A B D$ neurons in the experiments corresponds well to a mixture of the model $A B D_{M}$ and $A B D_{1}$ distributions.

Remarkably, the match between model predictions and experiments in Figure 4E involved fitting of only one free parameter. As mentioned above, the overall scale of model eye position sensitivities is arbitrary, and is related to the "read-out" of eye position from neural activity. This overall scale factor was adjusted to match model with experiment for the means of the VPNI distributions. The good match between the shapes of the VPNI distributions does not depend on any further parameter fitting. The same is true for the matches between the model and experimental distributions for the other neuron classes. There is one other adjustable parameter in the model, a scale factor in the weight matrix definition that was tuned to make the network operate as an integrator, but this scale factor is not otherwise involved in the prediction of eye position sensitivities.

To test the robustness of our result, we changed the model by adjusting the threshold for dividing nodes into center and periphery (Methods). Even if the number of neurons in the center was reduced by up to $50 \%$, the 

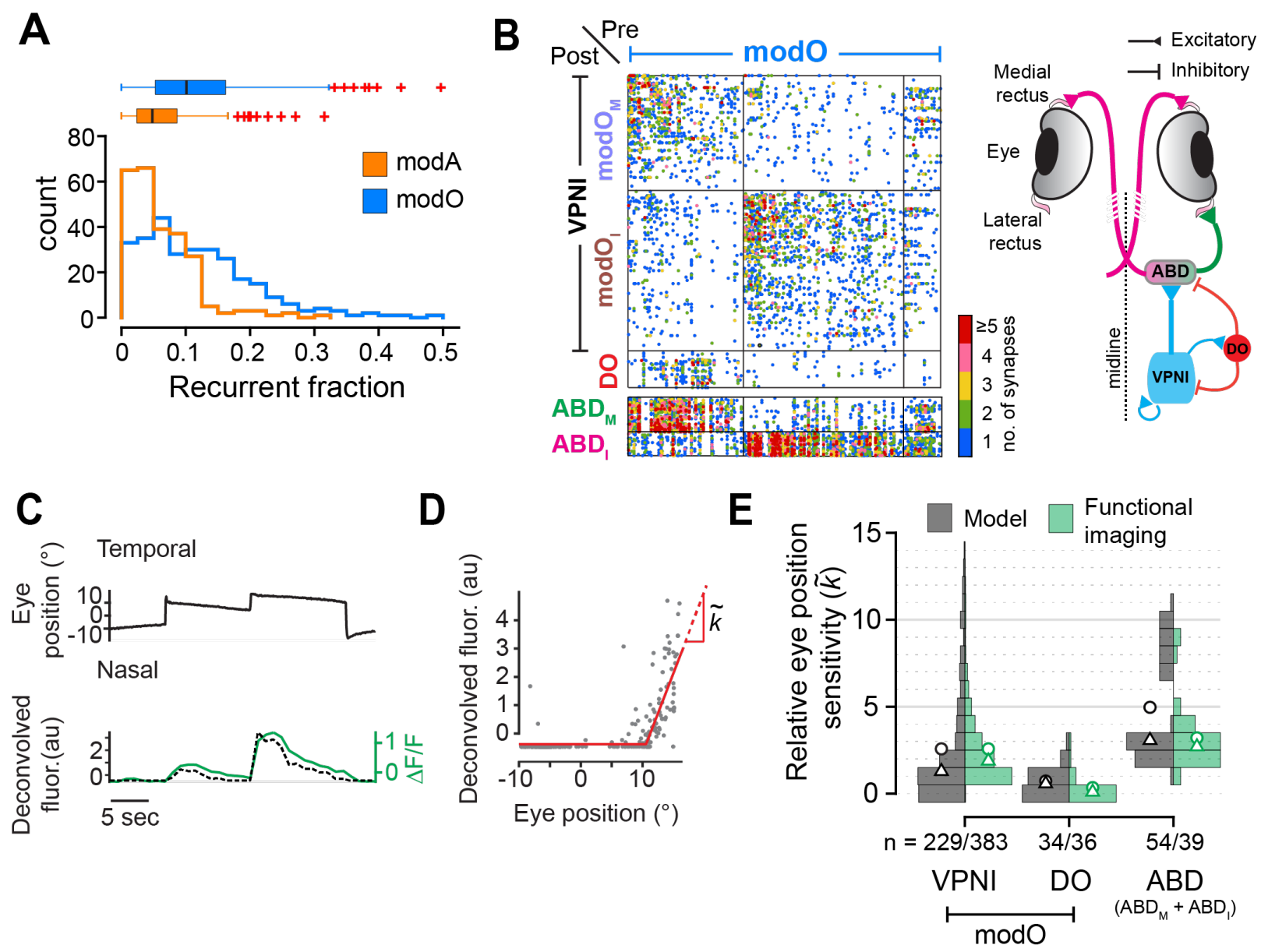

Figure 4: Connectome-based prediction of functional properties in the oculomotor system. A. Histogram of the fraction of all neurons that are recurrent within the identified modules. Box and whisker plots show medians (black line), $25^{\text {th }}$ and $75^{\text {th }}$ percentiles (box edges), and outliers (red +). B. (Left) Connectivity of neurons within modO, with DO neurons grouped separately for visualization. Connectivity from modO to ABD neurons in the periphery is plotted below. (Right) Schematic of a zebrafish summarizing connectivity between the different cell types, VPNI $\left(\operatorname{modO} \mathrm{M}_{\mathrm{M}}+\operatorname{modO}_{1}\right)$, $\mathrm{DO}$, and $\mathrm{ABD}\left(\mathrm{ABD_{M }}+\mathrm{ABD}_{\mathrm{I}}\right)$. C. Raw activity trace (green) from calcium imaging and the deconvolved fluorescence trace (dotted black line) of an example abducens neuron along with the eye position of the ipsilateral eye (black). D. For the neuron shown in (C), deconvolved fluorescence vs eye position (gray) and a best-fit relationship (red) that determines the relative eye position sensitivity $\tilde{k}$. E. Histograms of the relative eye position sensitivity $(\tilde{k})$ predicted from a connectome-based model (black) as compared to values determined from functional imaging experiments (green). Bimodal distribution of the ABD neurons in the model corresponds to $\mathrm{ABD}_{\mathrm{M}}$ (lower) and $\mathrm{ABD}_{\text {I }}$ (upper) populations. Circles represent the average values and triangles represent medians. Histograms for experimental data are showing values inside the $1^{\text {st }}$ and $99^{\text {th }}$ percentile. 
model distributions of eye position sensitivities remained similar (Figure S10A). We also corrupted our reconstructed wiring diagram by simulating errors in the automated synapse detection, and found that the population distributions of eye position sensitivities remained very similar (Figure S10C).

We wondered whether potential connectivity would have been sufficient for our network modeling. We estimated $N_{i j}$ by the number of potential synapses onto neuron $i$ from neuron $j$, and then computed the weight matrix $W_{i j}$ by the normalization described above (Methods). When our weight matrix based on potential synapses was substituted into our network model, we obtained population distributions for eye position sensitivities that were qualitatively different from experimental measurements (Figure S10D).

\section{Discussion}

Brain modules have previously been found by analyzing networks in which nodes represent brain regions (Zingg et al., 2014; Oh et al., 2014; Bota et al., 2015). We have instead considered networks in which nodes represent individual neurons, and links represent synaptic connections. The modules in our brainstem wiring diagram were validated using additional biological information, which also enabled plausible assignments of biological functions. A related study in visual cortex (Lee et al., 2016) did not yield modules that could be biologically validated or assigned functions. We proposed a hierarchical division (Figure 2, 3), but flat clustering yields similar modules (Figure S5).

When defined at cellular resolution, a module might sound similar to a neuronal cell type (Seung and Sümbül. 2014). The concepts have some relation but are different. The neurons of a module are strongly connected with each other by definition, whereas the neurons of a cell type might not be synaptically connected with each other at all.

We defined a potential synapse as a presynapse-postsynapse pair within some threshold distance, while the conventional definition is as an axodendritic apposition (Stepanyants and Chklovskii, 2005; Reimann et al. 2015). Our definition is convenient for EM, which reveals the locations of presynapses and postsynapses, but is also relevant for light microscopy if these synaptic structures are labeled. We found that some aspects of modular organization (Figs. 2D, 3C) and forward modeling of eye position sensitivities (Figure S10d) could be predicted from potential synapses if the threshold distance were $2 \mu \mathrm{m}$ or less. Based on this number, one might jump to the conclusion that at least some of our findings could have been obtained by diffraction-limited light microscopy. However, this technique usually requires the pooling of sparsely labeled neurons reconstructed from many animals (Shepherd et al., 2005; Jefferis et al., 2007), leading to two complications. First, spatial smoothing of arbors, typically over tens of microns, may be required to dampen random fluctuations in the number of potential synapses (Shepherd et al., 2005; Stepanyants and Chklovskii, 2005). Second, registration of multiple brains introduces additional positional uncertainty. Therefore it seems challenging to replace EM by diffraction-limited light microscopy, given that our model predictions become very poor or collapse when the threshold distance for potential synapses is $10 \mu \mathrm{m}$ (Figs. 20, 3C, Figure S10D). Novel light microscopies that beat the diffraction limit (Igarashi et al., 2018) could work in principle, but in practice have not so far yielded comparably rich information about neural circuits.

We identified modO as containing the velocity-to-position neural integrator (VPNI) of the oculomotor system. Previous physiological studies of VPNI cells mainly focused on R7/8 (Miri et al., 2011b; Lee et al., 2015; Daie et al., 2015; Vishwanathan et al., 2017). Our map of modO (Figure 2C) suggests that the VPNI should also include R4-6. The extension is consistent with a previous observation that VPNI function was only partially abolished by sizable inactivation of R7/8 (Miri et al., 2011b), and with previous reports of eye position signals in R4-6 neurons that are not abducens neurons (Ramirez and Aksay, 2018; Brysch et al., 2019).

The VPNI has served as a model system for understanding persistent neural firing (Major and Tank, 2004 Joshua and Lisberger, 2015). The VPNI also exhibits low-dimensional brain dynamics, which has been found to underlie a wide array of motor (Cannon and Robinson, 1987; Seung, 1996; Aksay et al., 2001), navigational 
(Blair and Sharp, 1995; Samsonovich and McNaughton, 1997; Burak and Fiete, 2006, 2009; Yoon et al., 2013; Kim et al., 2017; Turner-Evans et al., 2020) and cognitive functions (Romo et al., 1999; Miller et al., 2005; Daie et al., 2015; Inagaki et al., 2019). Twenty-five years ago, "line attractor" and "ring attractor" network models were proposed for low-dimensional neural dynamics in the oculomotor (Seung, 1996) and head direction systems (Skaggs et al., 1995; Zhang, 1996), respectively. Connectomic information from the Drosophila head direction system (Turner-Evans et al., 2020) is currently being used to inform ring attractor network models (Skaggs et al. 1995; Zhang, 1996). Our work similarly constrains attractor network models of the oculomotor system (Seung 1996; Fisher et al., 2013). This is the beginning of a trend in which connectomics will aid network modeling of low-dimensional neural dynamics, a general phenomenon that has been recognized in many brain regions and species (Yoon et al., 2013; Daie et al., 2015; Kim et al., 2017; Green et al., 2017; Vyas et al., 2020).

In most neural network models of brain function, the synaptic weight matrix has been regarded as the solution to an "inverse problem." Given the observed effects (neural activity and behavior), the modeler attempts to identify the unobserved cause (weight matrix). Connectomics offers the possibility of treating network modeling as more of a "forward problem." The forward approach has been feasible for small nervous systems, in which the weight matrix can be directly observed and completely mapped by synaptic physiology (Hartline, 1979).

A similar "forward" approach has been applied in dedicated sensory circuits. Wanner and Friedrich (Wanner and Friedrich, 2020) demonstrated how a connectome could be used to model whitening of odor representations in a vertebrate olfactory bulb (Wanner and Friedrich, 2020). An EM wiring diagram was used to constrain a model of orientation and direction selectivity in the Drosophila visual motion detection circuit (Tschopp et al., 2018), though fine-tuning by backpropagation learning was necessary. At a lower, "mesoscopic" level of resolution, inter-area projection maps in primates have been used to explain the temporal dynamics of cortical responses (Wang et al., 2020).

Our forward approach started from a synaptic weight matrix estimated from EM reconstruction, and succeeded in predicting the statistical distribution of relative eye position sensitivities for several neural populations measured by calcium imaging of animals during ocular fixations (Figure 4C). Some "inverse" aspects to our modeling remained, because we constrained ourselves to modeling eye movements in the absence of body movements and used known signs of connections (Lee et al., 2015; Pastor et al., 2019), physiological observations about the approximate linearity of oculomotor responses (Aksay et al., 2000, 2001) and independence of the bilateral halves of the circuit (Aksay et al., 2007b; Debowy and Baker, 2011).

Our success in modeling eye position sensitivities through a forward approach with minimal physiological constraints is perhaps surprising. This is especially so given our naive estimates of synaptic weights from the simple measure of number of synapses, simplified linear rate model neuron treatment of cell morphology, synaptic and intrinsic cellular biophysics, and our neglect of neuromodulation (Bargmann and Marder, 2013). Comparisons between model predictions and physiological data at the level of single cells, rather than populations, might require more sophisticated modeling of cell- and synapse-specific biophysics.

As more connectomes become available in other settings, it will be important to consider which physiological constraints need to be incorporated to make appropriate use of these powerful datasets. Answers to this question depend on many factors including the breadth of behaviors to be produced in a single model, the range of dynamics of the constituent components, and the degree to which the model is to produce quantitative versus qualitative matches to data. Nevertheless, we hope our work suggests how, when guided by knowledge of the various behaviors a circuit participates in, and appropriate physiological constraints gleaned from recordings and perturbations of activity, it may be possible in even more complex circuits to identify physiological modules whose function can be well understood using the connectome-based analysis and modeling approach taken here. 


\section{Author Contributions}

AV - Designed and conceptualized study, collected data, data interpretation, imaged EM data, performed registration, data analysis and wrote paper. ADR - collected light microscopic data, registered and analyzed LM data, data interpretation. JW - code for data generation and curation in eyewire, meshing, skeletonization, convnet inference. AS- computational modeling, RY - clustering algorithm comparisons, NK - Eyewire data assembly. DI - EM image assembly. NT - Synapses detection and partner assignment. KL - Boundary detection. IT Eyewire algorithms development. WMS- Eyewire algorithms development, Data manipulation software. CSJ Eyewire algorithms development, Eyewire system administration. CD, DB - Eyewire moderation, data curation. MSG - designed and conceptualized computational model, data interpretation, wrote the paper. ERFA - designed and conceptualized study, data interpretation, wrote the paper. HSS- designed and conceptualized study, data analysis, data interpretation, wrote the paper. EyeWirers - Neuron reconstruction online.

\section{Acknowledgement}

We thank Merlin Moore, Kyle Wille, Ryan Willie, Selden Koolman, Sarah Morejohn, Ben Silverman, Doug Bland, Celia David, Sujata Reddy, Anthony Pelegrino, Sarah Williams and Dan Visser for manual annotation and proofreading of neuron reconstructions, Amy Sterling for EyeWire management. We thank Will Wong for help with image data transformation for Eyewire and Alex Bae for help with skeletonization. We also thank Misha Tsodyks, David Kleinfeld, Carlos Brody, Misha Ahrens, Minoru Koyama, Aristides Arrenberg, Christian Brysch, Kayvon Daie, Vishwas Mishra, and Chanwoo Chun for their suggestions. ERFA, MSG, AV and HSS acknowledge support from R01 NS104926, R01 EY027036. ERFA and MSG acknowledge support from R01 EY021581, Simons Foundation Global Brain Initiative. ADR received support from K99 EY027017. HSS acknowledges support from NIH/NCI UH2 CA203710, ARO W911NF-12-1-0594, and the Mathers Foundation, as well as assistance from Google, Amazon, and Intel. HSS is grateful for support from the Intelligence Advanced Research Projects Activity (IARPA) via Department of Interior/Interior Business Center (Dol/IBC) contract number D16PC0005. The U.S. Government is authorized to reproduce and distribute reprints for Governmental purposes notwithstanding any copyright annotation thereon.

\section{Declaration of Interest}

HSS has financial interests in Zetta AI LLC. 


\section{References}

Aksay, E., Baker, R., Seung, H.S., Tank, D.W., 2000. Anatomy and discharge properties of Pre-Motor neurons in the goldfish medulla that have Eye-Position signals during fixations. J. Neurophysiol. 84, 1035-1049.

Aksay, E., Gamkrelidze, G., Seung, H.S., Baker, R., Tank, D.W., 2001. In vivo intracellular recording and perturbation of persistent activity in a neural integrator. Nat. Neurosci. 4, 184-193.

Aksay, E., Olasagasti, I., Mensh, B.D., Baker, R., Goldman, M.S., Tank, D.W., 2007a. Functional dissection of circuitry in a neural integrator. Nat. Neurosci. 10, 494-504.

Aksay, E., Olasagasti, I., Mensh, B.D., Baker, R., Goldman, M.S., Tank, D.W., 2007b. Functional dissection of circuitry in a neural integrator. Nat. Neurosci. 10, 494-504.

Allen, W.F., 1932. Formatio reticularis and reticulospinal tracts, their visceral functions and possible relationships to tonicity and clonic contractions. Journal of the Washington Academy of Sciences 22, 490-495.

Bae, J.A., Mu, S., Kim, J.S., Turner, N.L., Tartavull, I., Kemnitz, N., Jordan, C.S., Norton, A.D., Silversmith, W.M., Prentki, R., Others, 2018. Digital museum of retinal ganglion cells with dense anatomy and physiology. Cell 173, 1293-1306.

Baldwin, C.Y., William L White Professor of Business Administration Carliss Y Baldwin, Clark, K.B., Clark, K.B., 2000. Design Rules: The power of modularity. MIT Press.

Bargmann, C.I., 2012. Beyond the connectome: how neuromodulators shape neural circuits. Bioessays 34, 458-465.

Bargmann, C.I., Marder, E., 2013. From the connectome to brain function. Nat. Methods 10, 483-490.

Berger, D.R., Seung, H.S., Lichtman, J.W., 2018. VAST (volume annotation and segmentation tool): Efficient manual and Semi-Automatic labeling of large 3D image stacks. Front. Neural Circuits 12, 88.

Bhattacharyya, K., McLean, D.L., Maclver, M.A., 2017. Visual threat assessment and reticulospinal encoding of calibrated responses in larval zebrafish. Curr. Biol. 27, 2751-2762.e6.

Bianconi, G., 2009. Entropy of network ensembles. Phys. Rev. E Stat. Nonlin. Soft Matter Phys. 79, 036114.

Blair, H.T., Sharp, P.E., 1995. Anticipatory head direction signals in anterior thalamus: evidence for a thalamocortical circuit that integrates angular head motion to compute head direction. J. Neurosci. 15, 6260-6270.

Blondel, V.D., Guillaume, J.L., Lambiotte, R., others, 2008. Fast unfolding of communities in large networks. Journal of statistical .

Bogovic, J.A., Hanslovsky, P., Wong, A., Saalfeld, S., 2016. Robust registration of calcium images by learned contrast synthesis, in: 2016 IEEE 13th International Symposium on Biomedical Imaging (ISBI), pp. 1123-1126.

Bota, M., Sporns, O., Swanson, L.W., 2015. Architecture of the cerebral cortical association connectome underlying cognition. Proc. Natl. Acad. Sci. U. S. A. 112, E2093-101.

Briggman, K.L., Bock, D.D., 2012. Volume electron microscopy for neuronal circuit reconstruction. Curr. Opin. Neurobiol. 22, 154-161.

Briggman, K.L., Helmstaedter, M., Denk, W., 2011. Wiring specificity in the direction-selectivity circuit of the retina. Nature 471, 183-188. 
Brysch, C., Leyden, C., Arrenberg, A.B., 2019. Functional architecture underlying binocular coordination of eye position and velocity in the larval zebrafish hindbrain. BMC Biol. 17, 110.

Burak, Y., Fiete, I., 2006. Do we understand the emergent dynamics of grid cell activity? J. Neurosci. 26, 9352-4; discussion 9354.

Burak, Y., Fiete, I.R., 2009. Accurate path integration in continuous attractor network models of grid cells. PLoS Comput. Biol. 5, e1000291.

Cabrera, B., Torres, B., Pásaro, R., Pastor, A.M., Delgado-Garcia, J.M., 1992. A morphological study of abducens nucleus motoneurons and internuclear neurons in the goldfish (Carassius auratus). Brain Res. Bull. 28, 137144.

Cannon, S.C., Robinson, D.A., 1987. Loss of the neural integrator of the oculomotor system from brain stem lesions in monkey. J. Neurophysiol. 57, 1383-1409.

Cannon, S.C., Robinson, D.A., Shamma, S., 1983. A proposed neural network for the integrator of the oculomotor system. Biol. Cybern. 49, 127-136.

Chung, F., 2005. Laplacians and the cheeger inequality for directed graphs. Ann. Comb. 9, 1-19.

Daie, K., Goldman, M.S., Aksay, E.R.F., 2015. Spatial patterns of persistent neural activity vary with the behavioral context of short-term memory. Neuron $85,847-860$.

Debowy, O., Baker, R., 2011. Encoding of eye position in the goldfish horizontal oculomotor neural integrator. J. Neurophysiol. 105, 896-909.

Denk, W., Briggman, K.L., Helmstaedter, M., 2012. Structural neurobiology: missing link to a mechanistic understanding of neural computation. Nat. Rev. Neurosci. 13, 351-358.

Ding, H., Smith, R.G., Poleg-Polsky, A., Diamond, J.S., Briggman, K.L., 2016. Species-specific wiring for direction selectivity in the mammalian retina. Nature 535, 105-110.

Fisher, D., Olasagasti, I., Tank, D.W., Aksay, E.R.F., Goldman, M.S., 2013. A modeling framework for deriving the structural and functional architecture of a short-term memory microcircuit. Neuron 79, 987-1000.

Gahtan, E., Sankrithi, N., Campos, J.B., O’Malley, D.M., 2002. Evidence for a widespread brain stem escape network in larval zebrafish. J. Neurophysiol. 87, 608-614.

Giovannucci, A., Friedrich, J., Gunn, P., Kalfon, J., Brown, B.L., Koay, S.A., Taxidis, J., Najafi, F., Gauthier, J.L., Zhou, P., Khakh, B.S., Tank, D.W., Chklovskii, D.B., Pnevmatikakis, E.A., 2019. CalmAn an open source tool for scalable calcium imaging data analysis. Elife 8.

Gleich, D., 2006. Hierarchical directed spectral graph partitioning. Information Networks .

Gonçalves, P.J., Arrenberg, A.B., Hablitzel, B., Baier, H., Machens, C.K., 2014. Optogenetic perturbations reveal the dynamics of an oculomotor integrator. Front. Neural Circuits 8.

Green, J., Adachi, A., Shah, K.K., Hirokawa, J.D., Magani, P.S., Maimon, G., 2017. A neural circuit architecture for angular integration in drosophila. Nature 546, 101-106.

Hartline, D.K., 1979. Pattern generation in the lobster (panulirus) stomatogastric ganglion. II. pyloric network simulation. Biol. Cybern. 33, 223-236. 
Holland, P.W., Laskey, K.B., Leinhardt, S., 1983. Stochastic blockmodels: First steps. Soc. Networks 5, $109-137$.

Huang, K.H., Ahrens, M.B., Dunn, T.W., Engert, F., 2013. Spinal projection neurons control turning behaviors in zebrafish. Curr. Biol. 23, 1566-1573.

Igarashi, M., Nozumi, M., Wu, L.G., Cella Zanacchi, F., Katona, I., Barna, L., Xu, P., Zhang, M., Xue, F., Boyden, E., 2018. New observations in neuroscience using superresolution microscopy. J. Neurosci. 38, 9459-9467.

Inagaki, H.K., Fontolan, L., Romani, S., Svoboda, K., 2019. Discrete attractor dynamics underlies persistent activity in the frontal cortex. Nature 566, 212-217.

loffe, S., Szegedy, C., 2015. Batch normalization: Accelerating deep network training by reducing internal covariate shift arXiv:1502.03167.

Jarrell, T.A., Wang, Y., Bloniarz, A.E., Brittin, C.A., Xu, M., Thomson, J.N., Albertson, D.G., Hall, D.H., Emmons, S.W., 2012. The connectome of a decision-making neural network. Science 337, 437-444.

Jefferis, G.S.X.E., Potter, C.J., Chan, A.M., Marin, E.C., Rohlfing, T., Maurer, Jr, C.R., Luo, L., 2007. Comprehensive maps of drosophila higher olfactory centers: spatially segregated fruit and pheromone representation. Cell 128, 1187-1203.

Jia, Y., Shelhamer, E., Donahue, J., Karayev, S., Long, J., Girshick, R., Guadarrama, S., Darrell, T., 2014. Caffe: Convolutional architecture for fast feature embedding, in: Proceedings of the 22Nd ACM International Conference on Multimedia, ACM, New York, NY, USA. pp. 675-678.

Joshua, M., Lisberger, S.G., 2015. A tale of two species: Neural integration in zebrafish and monkeys. Neuroscience 296, 80-91.

Karrer, B., Newman, M.E.J., 2011. Stochastic blockmodels and community structure in networks. Phys. Rev. E Stat. Nonlin. Soft Matter Phys. 83, 016107.

Kim, J.S., Greene, M.J., Zlateski, A., Lee, K., Richardson, M., Turaga, S.C., Purcaro, M., Balkam, M., Robinson, A., Behabadi, B.F., Campos, M., Denk, W., Seung, H.S., EyeWirers, 2014. Space-time wiring specificity supports direction selectivity in the retina. Nature 509, 331-336.

Kim, S.S., Rouault, H., Druckmann, S., Jayaraman, V., 2017. Ring attractor dynamics in the drosophila central brain. Science 356, 849-853.

Kimura, Y., Okamura, Y., Higashijima, S.I., 2006. alx, a zebrafish homolog of Chx10, marks ipsilateral descending excitatory interneurons that participate in the regulation of spinal locomotor circuits. J. Neurosci. 26, 56845697.

Kingma, D.P., Ba, J., 2014. Adam: A method for stochastic optimization arXiv:1412.6980.

Lee, K., Turner, N., Macrina, T., Wu, J., Lu, R., Seung, H.S., 2019. Convolutional nets for reconstructing neural circuits from brain images acquired by serial section electron microscopy. Curr. Opin. Neurobiol. 55, 188-198.

Lee, K., Zung, J., Li, P., Jain, V., Seung, H.S., 2017. Superhuman accuracy on the SNEMI3D connectomics challenge. arXiv preprint arXiv:1706.00120 .

Lee, M.M., Arrenberg, A.B., Aksay, E.R.F., 2015. A Structural and Genotypic Scaffold Underlying Temporal Integration. J. Neurosci. 35, 7903-7920. 
Lee, W.C.A., Bonin, V., Reed, M., Graham, B.J., Hood, G., 2016. Anatomy and function of an excitatory network in the visual cortex. Nature

Major, G., Tank, D., 2004. Persistent neural activity: prevalence and mechanisms. Curr. Opin. Neurobiol. 14, 675-684.

Markram, H., Muller, E., Ramaswamy, S., Reimann, M.W., Abdellah, M., Sanchez, C.A., Ailamaki, A., AlonsoNanclares, L., Antille, N., Arsever, S., Kahou, G.A.A., Berger, T.K., Bilgili, A., Buncic, N., Chalimourda, A., Chindemi, G., Courcol, J.D., Delalondre, F., Delattre, V., Druckmann, S., Dumusc, R., Dynes, J., Eilemann, S., Gal, E., Gevaert, M.E., Ghobril, J.P., Gidon, A., Graham, J.W., Gupta, A., Haenel, V., Hay, E., Heinis, T., Hernando, J.B., Hines, M., Kanari, L., Keller, D., Kenyon, J., Khazen, G., Kim, Y., King, J.G., Kisvarday, Z., Kumbhar, P., Lasserre, S., Le Bé, J.V., Magalhães, B.R.C., Merchán-Pérez, A., Meystre, J., Morrice, B.R., Muller, J., MuñozCéspedes, A., Muralidhar, S., Muthurasa, K., Nachbaur, D., Newton, T.H., Nolte, M., Ovcharenko, A., Palacios, J., Pastor, L., Perin, R., Ranjan, R., Riachi, I., Rodríguez, J.R., Riquelme, J.L., Rössert, C., Sfyrakis, K., Shi, Y., Shillcock, J.C., Silberberg, G., Silva, R., Tauheed, F., Telefont, M., Toledo-Rodriguez, M., Tränkler, T., Van Geit, W., Díaz, J.V., Walker, R., Wang, Y., Zaninetta, S.M., DeFelipe, J., Hill, S.L., Segev, I., Schürmann, F., 2015. Reconstruction and simulation of neocortical microcircuitry. Cell 163, 456-492.

McFarland, J.L., Fuchs, A.F., 1992. Discharge patterns in nucleus prepositus hypoglossi and adjacent medial vestibular nucleus during horizontal eye movement in behaving macaques. J. Neurophysiol. 68, 319-332.

Metcalfe, W.K., Mendelson, B., Kimmel, C.B., 1986. Segmental homologies among reticulospinal neurons in the hindbrain of the zebrafish larva. J. Comp. Neurol. 251, 147-159.

Miller, P., Brody, C.D., Romo, R., Wang, X.J., 2005. A recurrent network model of somatosensory parametric working memory in the prefrontal cortex.

Miri, A., Daie, K., Arrenberg, A.B., Baier, H., Aksay, E., Tank, D.W., 2011a. Spatial gradients and multidimensional dynamics in a neural integrator circuit. Nature Publishing Group 14, 1150-1159.

Miri, A., Daie, K., Arrenberg, A.B., Baier, H., Aksay, E., Tank, D.W., 2011b. Spatial gradients and multidimensional dynamics in a neural integrator circuit. Nat. Neurosci. 14, 1150-1159.

Motta, A., Berning, M., Boergens, K.M., Staffler, B., Beining, M., Loomba, S., Hennig, P., Wissler, H., Helmstaedter, M., 2019. Dense connectomic reconstruction in layer 4 of the somatosensory cortex. Science 366.

Muggeo, V.M.R., 2003. Estimating regression models with unknown break-points. Stat. Med. 22, 3055-3071.

Nassim, C., 2018. Lessons from the Lobster: Eve Marder's Work in Neuroscience. MIT Press.

Newman, M., 2018. Networks. Oxford University Press.

Oh, S.W., Harris, J.A., Ng, L., Winslow, B., Cain, N., Mihalas, S., Wang, Q., Lau, C., Kuan, L., Henry, A.M., Mortrud, M.T., Ouellette, B., Nguyen, T.N., Sorensen, S.A., Slaughterbeck, C.R., Wakeman, W., Li, Y., Feng, D., Ho, A., Nicholas, E., Hirokawa, K.E., Bohn, P., Joines, K.M., Peng, H., Hawrylycz, M.J., Phillips, J.W., Hohmann, J.G., Wohnoutka, P., Gerfen, C.R., Koch, C., Bernard, A., Dang, C., Jones, A.R., Zeng, H., 2014. A mesoscale connectome of the mouse brain. Nature 508, 207-214.

Orger, M.B., Kampff, A.R., Severi, K.E., Bollmann, J.H., Engert, F., 2008. Control of visually guided behavior by distinct populations of spinal projection neurons. Nat. Neurosci. 11, 327-333.

Pastor, A.M., Calvo, P.M., de la Cruz, R.R., Baker, R., Straka, H., 2019. Discharge properties of morphologically identified vestibular neurons recorded during horizontal eye movements in the goldfish. J. Neurophysiol. 121, 1865-1878. 
Paszke, A., Gross, S., Chintala, S., Chanan, G., Yang, E., DeVito, Z., Lin, Z., Desmaison, A., Antiga, L., Lerer, A., 2017. Automatic differentiation in PyTorch.

Pavlovic, D.M., Vértes, P.E., Bullmore, E.T., Schafer, W.R., Nichols, T.E., 2014. Stochastic blockmodeling of the modules and core of the caenorhabditis elegans connectome. PLoS One 9, e97584.

Peixoto, T.P., 2014. Hierarchical block structures and High-Resolution model selection in large networks. Phys. Rev. X 4, 011047.

Pnevmatikakis, E.A., Soudry, D., Gao, Y., Machado, T.A., Merel, J., Pfau, D., Reardon, T., Mu, Y., Lacefield, C., Yang, W., Ahrens, M., Bruno, R., Jessell, T.M., Peterka, D.S., Yuste, R., Paninski, L., 2016. Simultaneous denoising, deconvolution, and demixing of calcium imaging data. Neuron 89, 285-299.

Pujala, A., Koyama, M., 2019. Chronology-based architecture of descending circuits that underlie the development of locomotor repertoire after birth. Elife 8.

Ramirez, A.D., Aksay, E.R.F., 2018. Pre-saccadic rise neurons in the hindbrain control the timing of spontaneous saccades.

Ramón-Moliner, E., Nauta, W.J., 1966. The isodendritic core of the brain stem: ISODENDRITIC CORE OF BRAIN STEM. J. Comp. Neurol. 126, 311-335.

Randlett, O., Wee, C.L., Naumann, E.A., Nnaemeka, O., Schoppik, D., Fitzgerald, J.E., Portugues, R., Lacoste, A.M.B., Riegler, C., Engert, F., Others, 2015. Whole-brain activity mapping onto a zebrafish brain atlas. Nat. Methods 12, 1039.

Reichardt, J., Bornholdt, S., 2006. Statistical mechanics of community detection. Phys. Rev. E Stat. Nonlin. Soft Matter Phys. 74, 016110.

Reimann, M.W., King, J.G., Muller, E.B., Ramaswamy, S., Markram, H., 2015. An algorithm to predict the connectome of neural microcircuits. Front. Comput. Neurosci. 9, 120.

Robinson, D.A., 1968. Eye movement control in primates. Science 161, 1219-1224.

Romo, R., Brody, C.D., Hernández, A., Lemus, L., 1999. Neuronal correlates of parametric working memory in the prefrontal cortex. Nature 399, 470-473.

Ronneberger, O., Fischer, P., Brox, T., 2015. U-Net: Convolutional networks for biomedical image segmentation arXiv:1505.04597.

Rubinov, M., Sporns, O., 2010. Complex network measures of brain connectivity: uses and interpretations. Neuroimage 52, 1059-1069.

Samsonovich, A., McNaughton, B.L., 1997. Path integration and cognitive mapping in a continuous attractor neural network model. J. Neurosci. 17, 5900-5920.

Sato, M., Bitter, I., Bender, M.A., Kaufman, A.E., Nakajima, M., 2000. TEASAR: tree-structure extraction algorithm for accurate and robust skeletons, in: Proceedings the Eighth Pacific Conference on Computer Graphics and Applications, pp. 281-449.

Schoonheim, P.J., Arrenberg, A.B., Del Bene, F., Baier, H., 2010. Optogenetic localization and genetic perturbation of saccade-generating neurons in zebrafish. J. Neurosci. 30, 7111-7120.

Seung, H.S., 1996. How the brain keeps the eyes still. Proc. Natl. Acad. Sci. U. S. A. 93, 13339-13344. 
Seung, H.S., 2009. Reading the Book of Memory: Sparse Sampling versus Dense Mapping of Connectomes. Neuron 62, 17-29.

Seung, H.S., Sümbül, U., 2014. Neuronal cell types and connectivity: lessons from the retina. Neuron 83, 1262-1272.

Seung, S., 2012. Connectome: How the Brain's Wiring Makes Us Who We Are. HMH.

Shepherd, G.M.G., Stepanyants, A., Bureau, I., Chklovskii, D., Svoboda, K., 2005. Geometric and functional organization of cortical circuits. Nat. Neurosci. 8, 782-790.

Skaggs, W.E., Knierim, J.J., Kudrimoti, H.S., McNaughton, B.L., 1995. A model of the neural basis of the rat's sense of direction, in: Advances in neural information processing systems, papers.nips.cc. pp. 173-180.

Sporns, O., Betzel, R.F., 2016. Modular brain networks. Annu. Rev. Psychol. 67, 613-640.

Stepanyants, A., Chklovskii, D., 2005. Neurogeometry and potential synaptic connectivity. Trends Neurosci. 28, 387-394.

Stepanyants, A., Hof, P.R., Chklovskii, D.B., 2002. Geometry and structural plasticity of synaptic connectivity. Neuron 34, 275-288.

Tschopp, F.D., Reiser, M.B., Turaga, S.C., 2018. A connectome based hexagonal lattice convolutional network model of the drosophila visual system arXiv:1806.04793.

Turner, N.L., Lee, K., Lu, R., Wu, J., Ih, D., Seung, H.S., 2020. Synaptic partner assignment using attentional voxel association networks, in: 2020 IEEE 17th International Symposium on Biomedical Imaging (ISBI), pp. $1-5$.

Turner-Evans, D.B., Jensen, K.T., Ali, S., Paterson, T., Sheridan, A., Ray, R.P., Wolff, T., Lauritzen, J.S., Rubin, G.M., Bock, D.D., Jayaraman, V., 2020. The neuroanatomical ultrastructure and function of a biological ring attractor. Neuron 108, 145-163.e10.

Turrigiano, G., 2012. Homeostatic synaptic plasticity: local and global mechanisms for stabilizing neuronal function. Cold Spring Harb. Perspect. Biol. 4, a005736.

Varshney, L.R., Chen, B.L., Paniagua, E., Hall, D.H., Chklovskii, D.B., 2011. Structural properties of the caenorhabditis elegans neuronal network. PLoS Comput. Biol. 7, e1001066.

Vishwanathan, A., Daie, K., Ramirez, A.D., Lichtman, J.W., Aksay, E.R.F., Seung, H.S., 2017. Electron Microscopic Reconstruction of Functionally Identified Cells in a Neural Integrator. Curr. Biol. 27, 2137-2147.e3.

Vyas, S., Golub, M.D., Sussillo, D., Shenoy, K.V., 2020. Computation through neural population dynamics. Annu. Rev. Neurosci. 43, 249-275.

Wagner, G.P., Pavlicev, M., Cheverud, J.M., 2007. The road to modularity. Nat. Rev. Genet. 8, 921-931.

Wang, X.J., Pereira, U., Rosa, M.G., Kennedy, H., 2020. Brain connectomes come of age. Curr. Opin. Neurobiol. $65,152-161$.

Wanner, A.A., Friedrich, R.W., 2020. Whitening of odor representations by the wiring diagram of the olfactory bulb. Nat. Neurosci. 23, 433-442.

Wu, J., Silversmith, W.M., Seung, H.S., 2019. Chunkflow: Distributed hybrid cloud processing of large 3D images by convolutional nets. arXiv preprint arXiv:1904.10489. 
Xu, C.S., Januszewski, M., Lu, Z., Takemura, S., Hayworth, K., others, 2020. A connectome of the adult drosophila central brain. bioRxiv .

Yoon, K., Buice, M.A., Barry, C., Hayman, R., Burgess, N., Fiete, I.R., 2013. Specific evidence of low-dimensional continuous attractor dynamics in grid cells. Nat. Neurosci. 16, 1077-1084.

Zhang, K., 1996. Representation of spatial orientation by the intrinsic dynamics of the head-direction cell ensemble: a theory. J. Neurosci. 16, 2112-2126.

Zingg, B., Hintiryan, H., Gou, L., Song, M.Y., Bay, M., Bienkowski, M.S., Foster, N.N., Yamashita, S., Bowman, I., Toga, A.W., Dong, H.W., 2014. Neural networks of the mouse neocortex. Cell 156, 1096-1111.

Zlateski, A., Sebastian Seung, H., 2015. Image segmentation by Size-Dependent single linkage clustering of a watershed basin graph arXiv:1505.00249.

Zung, J., Tartavull, I., Lee, K., Sebastian Seung, H., 2017. An error detection and correction framework for connectomics. Advances in Neural Information Processing Systems 30 (NIPS 2017) arXiv:1708.02599. 


\section{Methods}

\section{Image acquisition and alignment}

We acquired a dataset of the larval zebrafish hindbrain that extended $250 \mu \mathrm{m}$ rostrocaudally and includes rhombomeres 4 through $7 / 8$ (R4 to R7/8). The volume extends $120 \mu \mathrm{m}$ laterally from the midline and $80 \mu \mathrm{m}$ ventrally from the plane of the Mauthner cell axon. The ssEM dataset was an extension of the original dataset in ref Vishwanathan et al. (2017) and was extended by additional imaging of the same serial sections. Only a few tens of neurons had been manually reconstructed in our original publication on the ssEM dataset Vishwanathan et al. (2017). The dataset was stitched and aligned using a custom package, Alembic (see Code availability). The tiles from each section were first montaged in 2D, and then registered and aligned in 3D as whole sections. Point correspondences were generated by block matching via normalized cross-correlations both between tiles and across sections. The final set of parameters that were used are listed in table .

Table1: Parameters used for image alignment

\begin{tabular}{|c|c|c|c|c|c|c|c|}
\hline Step & Images & BlockMatches & Transformation & $\begin{array}{l}\text { Typical block } \\
\text { radius (pixels) }\end{array}$ & $\begin{array}{l}\text { Typical search } \\
\text { radius (pixels) }\end{array}$ & Scale & $\begin{array}{l}\text { Bandpass (after } \\
\text { downsampling) }\end{array}$ \\
\hline Premontage & Tiles $(8 \mathrm{k} \times 8 \mathrm{k})$ & 1 between each pair of adjacent tiles & Translation & Entire overlap & Entire overlap & 0.5 & $(0,10)$ \\
\hline Elastic Montage & Tiles (8k x 8k) & $\begin{array}{c}\text { Regular triangular mesh, } 100 \mathrm{px} \text {, in the } \\
\text { overlapping regions between adjacent } \\
\text { tiles }\end{array}$ & Elastic & 120 & 150 & 0.5 & $(0,20)$ \\
\hline Pre-Prealignment & sections & 1 between each adjacent section & Translation & $35 \%$ of images & entire images & 0.03 & \\
\hline Prealignment & sections & $\begin{array}{l}\text { Regular triangular mesh, } 2000 \mathrm{px} \text {, } \\
\text { between sections }\end{array}$ & Regularized affine & 800 & 3500 & 0.25 & $(2.5,12.5)$ \\
\hline Rough alignment & sections & $\begin{array}{c}\text { Regular triangular mesh, } 375 \mathrm{px}, \\
\text { between sections }\end{array}$ & Elastic & 500 & 300 & 0.25 & $(2.5,10)$ \\
\hline Fine alignment & sections & $\begin{array}{l}\text { Regular triangular mesh, } 100 \mathrm{px}, \\
\text { between section }\end{array}$ & Elastic & 300 & 70 & 1 & $(2.5,15)$ \\
\hline
\end{tabular}

Errors in each step were found by a combination of programmed flags (such as lower than expected correspondences, small search radius, large distribution of norms, or high residuals after mesh relaxation) and visual inspection. They were corrected by either changing the parameters or by manual filtering of points. In most cases, the template and the source were both passed through a band-pass filter. Stitching of tiles (montaging) within a single section was split into a linear translation step (premontage) and a non-linear elastic step (elastic montage). In the premontage step individual tiles were assembled to have $10 \%$ overlap between neighboring tiles, as specified during imaging, and by fixing a single tile (anchoring) in place. They were then translated row by row and column by column according to the single correspondence found between the overlaps. In the elastic montage step, the locations of the tiles were initialized from the translated locations found previously, and blockmatches were computed every 100 pixels on a regular triangular mesh (see Table for parameters used).

Once the correspondences were found, outliers were filtered by checking the spatial distribution of the crosscorrelogram (sigma filter), height of the peak of the correlogram ( $r$ value), dynamic range of the source patch contrast, kurtosis of the source patch, local consensus (average of immediate neighbors), and global consensus (inside the section). After the errors had been corrected, by filtering bad matches, the linear system was solved using conjugate gradient descent. The mean residual errors were in the range of $0.5-1.0$ pixels after relaxation. The inter-section alignment was split into a translation step (pre-prealignment), a regularized affine step (prealignment), a fast coarse elastic step (rough alignment), and a slow fine elastic step (fine alignment). In the pre-prealignment step, a central patch of the given montaged section was matched to the previous montaged section to obtain the rough translation between two montaged sections. In the prealignment step, the montaged images were offset by that translation, and then a small number of correspondences were found between the two montaged sections, which were solved for a least-squared-residual affine transform, regularized with $10 \%$ (empirically derived) of identity transformation to reduce shear from propagating across multiple sections. Proceeding sequentially allowed the entire stack to get roughly in place. The mean residual errors were in the range of 3.5 pixels after relaxation. 


\section{Convolutional Net Training}

\section{Dataset}

Four expert brain image analysts (Daan Visser, Kyle Willie, Merlin Moore, and Selden Koolman) manually segmented neuronal cell boundaries from six subvolumes of EM images with VAST (Berger et al., 2018), labeling 194.4 million voxels in total. These labeled subvolumes were used as the ground truth for training convolutional networks to detect neuronal boundaries. We used 187.7 million voxels for training and reserved 6.7 million voxels for validation.

\section{Network architecture}

To detect neuronal boundaries, we used a multiscale 3D convolutional network architecture similar to the boundary detector in (Zung et al., 2017). This architecture was similar to U-Net (Ronneberger et al., 2015), but with more pathways between scales. We augmented the original architecture of (Zung et al., 2017) with two modifications. First, we added a "lateral" convolution between every pair of horizontally adjacent layers (i.e. between feature maps at the same scale). Second, we used batch normalization (loffe and Szegedy, 2015) at every layer (except for the output layer). These two architectural modifications were found to improve boundary detection accuracy and stabilize/speed-up training, respectively. For more details, we refer the reader to the Supplementary Section A.1 and Figure S10 in (Zung et al., 2017).

\section{Training procedures}

We implemented the training and inference of our boundary detectors with the Caffe deep learning framework (Jia et al., 2014). We trained the networks on a single Titan X Pascal GPU. We optimized the binary cross-entropy loss with the Adam optimizer (Kingma and Ba, 2014), initialized with $\alpha=0.001, \beta 1=0.9, \beta 2=0.999$, and $\varepsilon=0.01$. The step size $\alpha$ was halved when the validation loss plateaued, three times during training at $135 \mathrm{~K}, 145 \mathrm{~K}$, and $175 \mathrm{~K}$ iterations. We used a single training example (minibatch of size 1) to compute gradients for each training iteration. The gradient for target affinities (the degree to which image pixels are grouped together) in each training example was reweighted dynamically to compensate for the high imbalance between target classes (i.e. low and high affinities). Specifically, we weighted each affinity inversely proportional to the class frequency, which was computed independently within each of the three affinity maps $(x, y$, and $z)$ and dynamically in each training example. We augmented training data using (1) random flips and rotations by $90^{\circ}$, (2) brightness and contrast augmentation, (3) random warping by combining five types of linear transformation (continuous rotation, shear, twist, scale and perspective stretch), and (4) misalignment augmentation (K. Lee et al. 2017) with the maximum displacement of 20 pixels in $\mathrm{x}$ - and $\mathrm{y}$-dimension. The training was terminated after 1 million iterations, which took about two weeks. We chose the model with the lowest validation loss at $550 \mathrm{~K}$ iterations.

\section{Convolutional Net Inference}

The above trained network was used to produce an affinity map of the whole dataset using the ChunkFlow.jl package (git link, (Wu et al., 2019)). Briefly, the computational tasks were defined in a JSON formatted string and submitted to a queue in Amazon Web Services Simple Queue Service (AWS SQS). We launched $13 \mathrm{com}-$ putational workers locally with NVIDIA TitanX GPU. The workers fetched tasks from the AWS SQS queue and performed the computation. The workers first cut out a chunk of the image volume using BigArrays.jl (git link) and decompose it into overlapping patches. The patches were fed into the convolutional network model to perform inference in PyTorch (Paszke et al., 2017). The output affinity map patches were blended in a buffer chunk. The output chunk was cropped around the margin to reduce boundary effects. The final affinity map chunk, which is 
aligned with block size in cloud storage, was uploaded using BigArrays.jl. Both image and affinity map volumes were stored in Neuroglancer precomputed format (https://neurodata.io/help/precomputed/). The inference took about 17 days in total and produced about 26 terabytes of affinity map.

\section{Chunk-wise Segmentation}

In order to perform segmentation of the entire volume, we divided the volume into 'chunks'. Overlapping affinity map chunks were cut out using BigArrays.jl, and a size-dependent watershed algorithm (Zlateski and Sebastian Seung, 2015) was applied to agglomerate neighboring voxels to make supervoxels. The agglomerated supervoxels are represented as a graph where the supervoxels are nodes, and the mean affinity values between contacting supervoxels are the edge weights. A minimum spanning tree was constructed from the graph by recursively merging the highest weight edges. This over-segmented volume containing all supervoxels and the minimum spanning tree was ingested into Eyewire (https://eyewire.org) for crowdsourced proofreading.

\section{Semi-automated reconstructions on Eyewire}

Neurons were chosen for proofreading in Eyewire based on an initial set of 'seed' neurons that were identified as carrying eye position signals, by co-registering the EM volume to calcium imaging performed on the same animal (Vishwanathan et al., 2017). All pre- and postsynaptic partners of the initial seed of 22 neurons were reconstructed. Following this we reconstructed partners of the neurons that were reconstructed in the initial round in a random manner. Eyewirers were provided the option of agglomerating (merging) supervoxels using a slider to change the threshold of agglomeration. To ensure accurate reconstructions, we did two things: (1) only players who met a certain threshold, determined by their accuracy on a previously published retinal dataset (Kim et al., 2014; Bae et al., 2018) were allowed to reconstruct zebrafish neurons and (2) the reconstructions were performed by two players in two rounds, in which the second player could modify the first player's reconstruction (Supplementary Information). Finally, after two rounds of reconstruction, neurons were validated by expert inhouse image analysts, who each have more than $5000 \mathrm{hrs}$ of experience. The resulting accuracy of the players in the crowd as compared to experts (assuming experts are 100\%) was $>80 \%$ in the first round and $~ 95 \%$ after the second round of tracing. The validated reconstructions were subsequently skeletonized for analysis purposes. Player accuracy was calculated as an F1 score, where F1 $=2 T P /(2 T P+F P+F N)$, where TP represents true positives, FP represents false positives, and FN represents false negatives. All scores were calculated as a sum over voxels. TP was assigned when both the player and the expert agreed the segmentation was correct. FN was assigned when the player missed segments that were added in by the expert. FP was assigned when the player erroneously added segments that did not belong. Two F1 scores were calculated for each player, once for round 1 and once for round 2 . No player played the same neuron in both rounds. Typically at an agglomeration threshold of 0.3 the segmentation had an F1 score of $62 \%$.

\section{Skeletonization}

The neuron segmentation IDs were ingested to an AWS SQS queue and multiple distributed workers were launched in Google Cloud using kubernetes. Each worker fetched the segmentation chunks associated with a neuron ID. The segmentation voxels were extracted as a point cloud and the Distance to Boundary Field (DBF) was computed inside each chunk. Finally a modified version of the skeletonization algorithm TEASAR was applied (Sato et al., 2000). Briefly, we constructed a weighted undirected graph from the point cloud, where the neighboring points are connected with an edge and the edge weight is computed from the DBF. Then, we took the point with the largest DBF as source, and found the furthest point as the target. The shortest path from source to target in the graph was computed as the skeleton nodes. The surrounding points were labeled as visited, and the closest remaining unvisited point was taken as the new source. We repeated this process until all the points 
were visited. The skeleton node diameter was set as its DBF. The skeleton nodes were post-processed by removing redundant nodes, removing 'hairs', based on diameter, removing branches inside soma, downsampling the nodes, merging single-child segments, and smoothening the skeleton path. All skeletonization was performed at MIP level 4

\section{Synapse detection}

Synapses were automatically segmented in this dataset using neural networks to detect clefts and assign the correct partner as previously described (Turner et al., 2020). Briefly, a subset of the imaged data $\left(219 \mu \mathrm{m}^{3}\right)$ was selected for annotation. The annotations were performed using the manual annotation tool VAST (Berger et al., 2018). Trained human annotators labeled the voxels that were part of the postsynaptic density (PSD) and presynaptic docked vesicle pools. A convolutional neural network was trained to match the PSD, using $107 \mu \mathrm{m}^{3}$ as a training set, and $36 \mu \mathrm{m}^{3}$ as a validation set, leaving the remaining $76 \mu \mathrm{m}^{3}$ as an initial test set. All of these sets were compared to the predictions of the model tuned to an F-Score of 1.5 on the validation set in order to bias towards recall, where recall $=T P /(T P+F N)$. Biasing the predictor towards recall reduces false negatives at the cost of more false positives, which are easier to correct. Apparent human errors were corrected, and training was restarted with a new model. We also later expanded the test set by proofreading similar automated results applied to new sections of the datasets (to increase representation of rare structures in the full image volume). The final model used a RS-UNet architecture (Lee et al., 2017) implemented using PyTorch (Paszke et al., 2017), and was trained using a manual learning rate schedule, decreasing the rate by a factor of 10 when the smoothed validation error converged. The final network reached $86 \%$ precision and $83 \%$ recall on the test set after $230 \mathrm{k}$ training iterations.

A convolutional network was also trained to assign synaptic partners to each predicted cleft as previously described (Turner et al., 2020). All 361 synapses in the ground truth were labeled with their synaptic partners, and the partner network used 204 synapses as a training set, 73 as a validation set, and the remaining 84 as a test set. The final network was $95 \%$ accurate in assigning the correct partners of the test set after $380 \mathrm{k}$ training iterations.

The final cleft network was applied across the entire image volume, and formed discrete predictions of synaptic clefts by running a distributed version of connected components. Each cleft was assigned synaptic partners by applying the partner network to each predicted cleft within non-overlapping regions of the dataset $(1024 \times 1024$ x 1792 voxels each). In the case where a cleft spanned multiple regions, the assignment within the region that contained the most of that cleft was accepted, and the others were discarded. Cleft regions whose centroid coordinates were within $1 \mu \mathrm{m}$ and were assigned the same synaptic partners were merged together in order to merge artificially split components.

Finally, spurious synapse assignments (i.e postsynapses on axons and presynapses on dendrites) were cleaned by querying the identity of the 10 nearest synapses to every synapse, where each synapse was associated with its closest skeleton node on both the pre- and post-synaptic sides. If the majority of the 10 nearest neighbors were of the same identity (pre or post), then the synapse was assigned correctly. If the majority were of an opposing identity, these synapses were assigned wrongly and were deleted. This process eliminated 1975 falsely assigned synapses ( 2\% of the total).

\section{Registration to reference atlas}

Registration of the EM dataset to the Z-Brain reference atlas (Randlett et al., 2015) was carried out in two stages. We created an intermediate EM stack from the low resolution (270 nm/pixel) EM images of the entire larval brain tissue. This intermediate stack had the advantage of a similar field of view as compared to the LM reference volume, while also being of the same imaging modality as the high-resolution EM stack. The low-resolution EM stack was registered to the reference brain by fitting an affine transform that maps the entire EM volume onto the 
LM volume. To do this, we selected corresponding points such as neuronal clusters and fiber tracts using the tool BigWarp (Bogovic et al., 2016). These corresponding points were used to determine an affine transform using the MATLAB least squares solver (mldivide). Subsequently, the intermediate EM stack, in the same reference frame as the Z-Brain atlas, was used as the template to register the high-resolution EM stack onto it. This was performed in a similar manner by selecting corresponding points and fitting an affine transform. The resulting transform would transform points from the high-resolution EM space to the reference atlas space. This transform was used to map the reconstructed skeletons from high-resolution EM space to the reference atlas space.

\section{Identification of ABD neurons}

We identified abducens motor neurons $\left(A B D_{M}\right.$, Figure $1 \mathrm{C}$, Figure $\left.\mathrm{S} 3\right)$ by their overlap with the mnx transgenic line (S3A). ABD $M$ axons exited R5 and R6 through the abducens $\left(\mathrm{VI}^{\text {th }}\right.$ ) nerve (Figure $1 \mathrm{C}$, black box) as reported previously (Vishwanathan et al., 2017). Contraversive horizontal movements of the eye are driven by the medial rectus muscle, which are innervated by motor neurons in the oculomotor nucleus, which in turn are driven by internuclear neurons $\left(A B D_{l}\right)$ in the contralateral abducens complex (Figure $\left.1 \mathrm{C}\right)$ ). $A B D_{1}$ neurons were identified (Figure $\mathrm{S} 3 \mathrm{~A}$ ) by their overlap with two nuclei in the evx2 transgenic line that labels glutamatergic interneurons. The $A B D_{1}$ neurons were just dorsal and caudal to the $A B D_{M}$ neurons, and their axons crossed the midline (Cabrera et al. 1992).

\section{Identification of DO neurons}

We identified a class of secondary vestibular neurons known as Descending Octavolateral (DO) neurons (Figure $11 \mathrm{D}$, brown). We observed that DO cells received synapses from primary vestibular afferents. The latter were orphan axons in R4 identified as the vestibular branch of the vestibulocochlear nerve (VIII ${ }^{\text {th }}$ nerve) by comparison with the isl-2 line, which labels the major cranial nerves (Figure S3B, blue axons).

\section{Identification of Reticulospinal neurons}

The RS neurons were divided into large and small groups (Figure1 1 ). Large RS neurons were the M, Mid2, MiM1, Mid3i and CaD neurons. Small RS neurons were RoV3, MiV1, MiV2. These were identified by their stereotypic locations (Metcalfe et al., 1986) and by comparison within the Z-Brain atlas (Figure S4).

\section{Centrality-based division into center and periphery}

The division of the reconstructed wiring diagram into center and periphery is based on standard measures of "centrality" which have been devised in network science (Newman, 2018). We define the simplest measure, known as degree centrality, as the geometric mean of the in-degree and out-degree of a node. (It is more common to choose one or the other.) Another popular measure, known as eigenvector centrality or eigencentrality, is a node's element in the eigenvector of the connection matrix $N_{i j}$ (number of synapses onto node $i$ from node $j$ ) corresponding to the eigenvalue with maximal real part--this measure extends the simpler concept of degree centrality by weighing a node's connections by their centrality, i.e. a node is more central to the network if it receives inputs from other high centrality neurons. Mathematically, this defines the eigenvector problem $\nu_{i}=$ $\Sigma N_{i j} \nu_{j}$ where $\nu_{i}$ is the (input) centrality of neuron i. An analogous formula, but instead replacing $N_{i j}$ by its transpose (thus, defining left rather than right eigenvectors) can be used to weight output connections by the (output) centrality of the node to which output is projected. The eigenvector elements can be chosen non-negative by the Perron-Frobenius theorem. It is standard to use either the left or right eigenvector, but we use both for our definition by computing the geometric mean of the left and right eigenvector elements. Degree centrality 
and eigencentrality are correlated, but not perfectly (Figure S1C). For a visualization of the network based on eigencentrality, see Figure S1E.

Our definition of the periphery relies mainly on degree centrality; the vast majority of the periphery consists of 2282 nodes with vanishing degree centrality. We also define the periphery to include an additional 62 nodes with vanishing $\left(<10^{-8}\right)$ eigencentrality but nonzero degree centrality. The remaining 540 recurrently connected neurons are defined as the "center" of the graph. (See below for effects of varying the eigencentrality threshold for center-periphery division.)

\section{Graph Clustering}

We applied three graph-clustering algorithms to divide the center into modules, and obtained similar results from all three. The clustering from the Louvain algorithm is presented in the main text, and those of the spectral algorithm and stochastic block model in the supplementary information .

\section{Louvain Clustering}

Graph clustering was performed using the Louvain clustering algorithm for identifying different 'communities' or 'modules' in an interconnected network by optimizing the 'modularity' of the network, where modularity measures the (weighted) density of connections within a module compared to between modules. Formally, the modularity measure maximized is $Q_{g e n}=\sum B_{i j} \delta\left(c_{i}, c_{j}\right)$, where $\delta\left(c_{i}, c_{j}\right)$ equals 1 if neurons a and $\mathrm{b}$ are in the same module and 0 otherwise, where $B_{i j}=\frac{1}{\omega}\left(W_{i j}-\gamma \frac{s_{i} s_{j}}{\omega}\right)+\operatorname{transpose}(B)$. Here $s_{i}=\sum_{c} W_{i c}$ is the sum of weights into node $i$, $s_{j}=\sum_{c} W_{j b}$ is the sum of weights out of node $j, \omega=\sum_{c d} W_{c d}$ is the total sum of weights in the network, and the resolution parameter $\gamma$ determines how much the naively expected weight of connections $\gamma \frac{s_{i} s_{j}}{\omega}$ is subtracted from the connectivity matrix. Potential degeneracy in graph clustering was addressed by computing the consensus of the clustering similar to (Sporns and Betzel, 2016). Briefly, an association matrix, counting the number of times a node (neuron) is assigned to a given module, was constructed by running the Louvain algorithm 200 times. Next, a randomized association matrix was constructed by permuting the module assignment for each node. Reclustering the thresholded association matrix, where threshold was the maximum element of the randomized association matrix, provided consensus modules. We used the commuinity_louvain.m function from the Brain Connectivity Toolbox package (BCT, https://sites.google.com/site/bctnet/Home). In addition to the Louvain graph-clustering algorithm, we also clustered the 'center' with two alternate graph-clustering algorithms; spectral clustering and stochastic block matching, described below.

\section{Spectral Clustering}

We employed a generalized spectral clustering algorithm for weighted directed graphs to bisect the zebrafish 'center' subgraph as proposed by (Chung, 2005). Given a graph $G(V, E)$ and its weighted adjacency matrix $A \in \mathbb{R}_{>0}^{n \times n}$, where $A_{i j}$ indicates the number of synapses from neuron $i$ to neuron $j$, one can construct a Markov chain on the graph with a transition matrix $P_{\alpha}$, such that $\left[P_{\alpha}\right]_{i j}:=(1-\alpha) \cdot A_{i j} / \sum_{k} A_{i k}+\alpha / n$. The coefficient $\alpha>0$ ensures that the constructed Markov chain is irreducible, and the Perron-Frobenius theorem guarantees $P_{\alpha}$ has a unique positive left eigenvector $\pi$ with eigenvalue 1 , where $\pi$ is also called the stationary distribution. The normalized symmetric Laplacian of the Markov chain is $\mathcal{L}=I-\frac{1}{2}\left(\Pi^{1 / 2} P_{\alpha} \Pi^{-1 / 2}+\Pi^{-1 / 2} P_{\alpha}^{\top} \Pi^{1 / 2}\right)$.

To approximately search for the optimal cut, we utilize the Cheeger inequality for a directed graph Chung (2005) that bridges the spectral gap of $\mathcal{L}$ and the Cheeger constant $\phi^{*}$. As shown in (Gleich, 2006), the eigenvector $v$ corresponding to the second smallest eigenvalue of $\mathcal{L}, \lambda_{2}$, results in optimal clusters. We obtained two clusters by a binary rounding scheme, i.e., $S=\left\{i \in V \mid v_{i} \geq 0\right\}$ and $S=\left\{i \in V \mid v_{i}<0\right\}$.

We modified the directed_laplacian_matrix function in the NetworkX package (https://networkx.github.io) to calculate the symmetric Laplacian for sparse connectivity matrices, with a default $\alpha=0.05$. The spectral gaps 
for the eigenvector-centrality subgraph is $\lambda_{2}^{\text {eigen }}=0.137$ and for the partitioned oculomotor $(\operatorname{modO})$ module is $\lambda_{2}^{\text {eigen }}=0.256$.

\section{Degree-corrected Stochastic Block Matching (SBM)}

Unlike the Louvain and spectral clustering algorithms that assume fewer intra-cluster connections than intercluster connections, the stochastic block models (SBMs) do not make this assumption. We applied an efficient statistical inference algorithm (Peixoto, 2014) to obtain the SBMs that best describes the 'center' subgraph.

The traditional SBM (Holland et al., 1983) is composed of $n$ vertices, divided into $B$ blocks with $\left\{n_{r}\right\}$ vertices in each block, and with the probability, $p_{r s}$, that an edge exists from block $r$ to block $s$. Here we use another equivalent definition, to use average edge counts from the observation $e_{r s}=n_{r} n_{s} p_{r s}$ to replace the probability parameters. The degree-corrected stochastic block model (Karrer and Newman, 2011) further specifies the inand out-degree sequences $\left\{k_{i}^{+}, k_{i}^{-}\right\}$of the graph as additional parameters.

To infer the best block membership $\left\{b_{i}\right\}$ of the vertices in the observed graph $G$, we maximize likelihood $\mathcal{P}\left(G \mid\left\{b_{i}\right\}\right)=1 / \Omega\left(\left\{e_{r s}\right\},\left\{n_{r}\right\}\right)$, where $\Omega\left(\left\{e_{r s}\right\},\left\{n_{r}\right\}\right)$ is the total number of different graph realizations with the same degree distribution $\left\{k_{i}^{+}, k_{i}^{-}\right\}$, and $\left\{e_{r s}\right\}$ edges among and within blocks of sizes $\left\{n_{r}\right\}$, corresponding to the block membership $\left\{b_{i}\right\}$. Therefore, maximizing likelihood is equivalent to minimizing the microcanonical entropy (Bianconi, 2009) $\mathcal{S}\left(\left\{e_{r s}\right\},\left\{n_{r}\right\}\right)=\ln \Omega\left(\left\{e_{r s}\right\},\left\{n_{r}\right\}\right)$, which can be calculated as $S \simeq-M-\sum_{i} \ln \left(k_{i}^{+} !\right)-$ $\sum_{i} \ln \left(k_{i}^{-} !\right)-\sum_{r s} e_{r s} \ln \left(\frac{e_{r s}}{\sum_{s} e_{r s} \sum_{r} e_{r s}}\right), \simeq-M-\sum_{i} \ln \left(k_{i}^{+} !\right)-\sum_{i} \ln \left(k_{i}^{-} !\right)-\sum_{r s} e_{r s} \ln \left(\frac{e_{r s}}{\sum_{s} e_{r s} \sum_{r} e_{r s}}\right), S$, where $M=\sum_{r s} e_{r s}$ is the total number of edges.

We used the minimize_blockmodel_dl function in the graph-tool package (https://graph-tool.skewed.de) to bisect the central subgraphs by setting $\bar{B}_{\min }=B_{\max }=2$ and degcorr $=$ true.

\section{Potential Synapse Formalism}

We define a potential synapse as a presynapse-postsynapse pair within a certain threshold distance (Figure 2D). This definition is somewhat different from the light microscopic approach, which defines a potential synapse (Stepanyants et al., 2002; Stepanyants and Chklovskii, 2005) as an approach of an axon and dendrite within some threshold distance. Also we use neurons reconstructed from a single animal, while the light microscopic approach aggregates neurons from multiple animals, or "clones" a single neuron many times Markram et al. (2015).

\section{Calcium imaging and eye position signals}

The complete methods for recording calcium activity used to create the functional maps are reported in (Ramirez and Aksay, 2018). Briefly, we used two-photon, raster-scanning microscopy to image calcium activity from single neurons throughout the hindbrain of 7-8 day old transgenic larvae expressing nuclear-localized GCaMP6f, $\mathrm{Tg}(\mathrm{HuC}$ :GCaMP6f-H2B) strain cy73-431 from Misha Ahrens' lab. Horizontal eye movements were recorded simultaneously with calcium signals using a substage CMOS camera. We used the CalmAn-Matlab software to extract the neuronal locations from fluorescence movies (Giovannucci et al., 2019).

We analyzed saccade-triggered average (STA) activity to determine which neurons were related to eye movements (see (Ramirez and Aksay, 2018) for complete details). For each cell, we interpolated fluorescence activity that occurred within five seconds before or after saccades to a grid of equally spaced, $1 / 3$ second timepoints and then averaged the interpolated activity across saccades to compute the STA. Separate STAs were taken for saccades towards the left and right. We performed a one-way ANOVA on each STA to determine which neurons had significant saccade-triggered changes in average activity $(p<0.01$ using the Holm-Bonferroni method to correct for multiple comparisons). To determine which of these neurons had activity related to eye movement and eye 
velocity, we first performed a Principal Components Analysis (PCA) on the STAs from neurons with significant saccade-triggered changes. We found that the first and second principal components had post-saccadic activity largely related to eye movement and eye velocity sensitivity respectively (see Figure 3A in (Ramirez and Aksay, 2018)). We characterized each STA using a scalar index called $\phi$ in (Ramirez and Aksay, 2018), created from that STA's projections onto the first two principal components and found that this index does a good job of characterizing the average eye movement and eye velocity-related activity seen across the population (see Figure $3 \mathrm{C}$ in (Ramirez and Aksay, 2018) for a map of values and population average STAs). Eye position and eye velocity neurons were defined as neurons with an STA whose value of $\phi$ was within a specific range (-83 to 37 and $38-$ 68 respectively). We removed any neurons with pre-saccadic activity that was significantly correlated with time until the upcoming saccade. The locations of each neuron were then registered to the Z-Brain atlas (Randlett et al., 2015) using similar methods as listed in the previous section (see (Ramirez and Aksay, 2018) for complete details).

\section{Relationship between firing rate and eye position}

To assess the functional characteristics of various oculomotor neurons (Figure 4) we fit a traditional model of eye position sensitivity to neuronal firing rates extracted from our fluorescence measurements. We approximated the relative firing rate, $r$, of a cell using the deconvolution algorithm with non-negativity constraint described in Pnevmatikakis et al. (2016). Comparison of relative firing rate across neurons in different populations was justified as we observed similar sensor expression levels and baseline noise levels in these populations.

We modeled the dependence of firing rate on eye position using the equation, $r=\left[\tilde{k}\left(E-E_{t h}\right)\right]_{+}$, where $\tilde{k}$ is the relative eye position sensitivity, $E_{t h}$ is the threshold eye position at which $r$ becomes positive, and the function $[x]_{+}=x$ if $x>0$ and 0 otherwise (Aksay et al., 2007a). In order to best compare results across animals, we normalized the units of eye position before fitting the model by subtracting the median eye position about the Null position (measured as the average raw eye position) and then dividing by the 95th percentile of the resulting positions. Since our focus was on a cell's position-dependence, we also eliminated the eye velocity dependent burst of spiking activity at the saccade time that ABD and VPNI neurons are known to display by removing samples that occur within 1.5 seconds before or 2 seconds after each saccade. Saccade times were found in an automated fashion by determining when eye velocity crossed a threshold value (Ramirez and Aksay, 2018). Finally, since the eye position and fluorescence were recorded at different sampling rates, we linearly interpolated the values of neuronal activity at the eye position sample times.

To fit the value of $\tilde{k}$, for each cell, we defined the eye movements toward the cell's responsive direction as positive so that $\tilde{k}$ is positive by construction. We then determined the threshold, $E_{t h}$, using an iterative estimation technique based on a Taylor series approximation of $[x]+$ described in Ref. (Muggeo, 2003). Using the resulting estimate of $E_{t h}$, we determined $\tilde{k}$ as the slope resulting from a linear regression (with offset) to $r$ using $E$ as a regressor. Since we do not know the cell's responsive direction a priori, we ran the model twice -- once with movements to the left as positive and once with movements to the right as positive -- and used the value of $\tilde{k}$ that resulted in the highest $R^{2}$ value.

As a goodness-of-fit measure we required all neurons, except DO neurons, to have an $R^{2}$ value greater than 0.4. Additionally, non-DO neurons were required to have a saccade-triggered average with at least one significant time point ( $p<0.01$ by an ANOVA test using Holm-Bonferroni correction) as defined in (Ramirez and Aksay, 2018) and to have a $d F / F$ response that was loosely related to eye position ( $R^{2}$ greater than 0.2 when we run the above model replacing $r$ with $d F / F$ ). The relative eye position sensitivity, $\tilde{k}$, for fluorescence data was then scaled to average physiological VPNI responses from goldfish (Aksay et al., 2007a; Debowy and Baker, 2011). 


\section{Identification of excitatory versus inhibitory neurons}

VPNI neurons in the dorsomedial stripe running from R4 to R7/8 overlap in the Z-Brain atlas with a region of alx expression (Figure. S9C, ZBB-alx-gal4) where most neurons are glutamatergic (Kimura et al., 2006). More lateral and caudal neurons overlap with markers for glycine and glutamate. Finally, VPNI neurons (which have ipsilateral axonal projections) in the oculomotor module do not overlap with the GABA expression (Figure S9C, $Z B B$-gad1b). For these reasons we assigned neurons in the oculomotor module to be excitatory. DO neurons with ipsilateral projections (used in our unilateral model) have been identified as being inhibitory (Pastor et al. 2019).

\section{Network model based on synaptic wiring diagram}

A unilateral model of the oculomotor integrator was built using the reconstructed synapses for the ABD, DO, and VPNI populations. Although the VPNI is a bilateral circuit, previous experiments (Aksay et al., 2007a; Debowy and Baker, 2011) have shown that one half of the VPNI is nevertheless capable of maintaining the ipsilateral range of eye positions after the contralateral half of the VPNI is silenced. This may reflect that most neurons in the contralateral half are below a threshold for transmitting synaptic currents to the opposite side when the eyes are at ipsilateral positions (Fisher et al., 2013). Therefore, we built a recurrent network model of one half of the VPNI circuit based on the modO neurons that we had reconstructed from one side of the zebrafish brainstem. We did not include the modA neurons, assuming that input from modA cells fell below a threshold needed to drive modO neurons, similar to the manner in which bilateral interactions have been shown to be negligible for the maintenance of persistent activity. We added the ABD neurons and the feedforward connections from modO to $A B D$ to the model because the ABD neurons are the "read-out" of the oculomotor signals in the VPNI. Projections from the DO population were taken to be inhibitory while all other connections were taken to be excitatory, as explained above. Remaining neurons in the oculomotor module were included as part of the VPNI--we did not consider other vestibular populations since only DO neurons in modO received vestibular afferents from the $\mathrm{VIII}$ th nerve; saccadic burst neurons and recently discovered pre-saccadic ramp neurons are likely located in R2/3 (Ramirez and Aksay, 2018), outside of our reconstructed volume.

Directed connection weights between each pair of neurons were set in proportion to the number of synapses from the presynaptic neuron onto the postsynaptic neuron divided by the total number synapses onto the postsynaptic neuron $W_{i j}= \pm \beta \frac{N_{i j}}{\Sigma_{k} N_{i k}}$. Thus, we assume that each element $W_{i j}$ corresponds to the fraction of total inputs to neuron $i$ that are provided by neuron $j$. The scale factor $\beta$ was set to achieve perfect integration in a linear rate model governed by $\tau \frac{d r_{i}}{d t}=-r_{i}+W_{i j} r_{j}$, where $r_{i}$ is the firing rate of the $i^{t h}$ neuron, $W_{i j}$ are the connection weights, and $\tau$, the intrinsic time constant, is $100 \mathrm{~ms}$. Fine tuning of this scale factor to achieve perfectly stable fixations is not critical to the results obtained here as the time courses of the persistent neural activity does not affect the relative firing rates of neurons that determine their relative eye position sensitivities. Position sensitivities could be determined numerically by simulating the response of the network to a pulse of input along the integrating direction. However, for a linear network, this is not necessary because the resulting relative persistent firing rates are equal to the leading eigenvector of the matrix $W_{i j}$ and thus can be determined analytically. Finally, the position sensitivity was multiplied by a single global scale factor that was determined by matching the average VPNI population responses from the model to the average physiological VPNI responses from goldfish (Aksay) et al., 2007a; Debowy and Baker, 2011).

As a test of the robustness of our results to possible errors in connectome reconstruction, we generated a connectome that accounted for the estimated false positive and negative rate of synapse detection by our connectome reconstruction procedure. We generated 1000 models by randomly varying the identified synapses according to the estimated false positive and false negative rates and calculated the connection weights as described above. The eye position sensitivities with this synaptic detection jitter were reported as the average of these 1000 models (Figure S10C). We also tested how robust our results were to the cutoff criterion for including neurons 
in the recurrently connected center by progressively increasing the minimum eigenvector centrality criterion for counting a neuron as belonging to the center as opposed to periphery. We then plotted how the simulation model results changed as a function of the number of center neurons was decreased and simultaneously reported the resulting number of VPNI (i.e. non-DO neurons in modO) neurons (Figure S10A). To characterize the degradation in model performance when the actual connectome was replaced by connectomes generated by spatial proximity (potential connections), we re-ran all of the analyses described above using potential connectomes defined for connections within 2,5 , or $10 \mu \mathrm{m}$.

\section{Code availability}

- Alembic - https://github.com/seung-lab/Alembic.git

- BigArrays.jl - https://github.com/seung-lab/BigArrays.jl with Apache License Version 2.0.

- ChunkFlow.jl - https://github.com/seung-lab/ChunkFlow.jl with Apache License Version 2.0.

- Watershed - https://github.com/seung-lab/Watershed.jl with GNU General Public License v3.0.

- Agglomeration - https://github.com/seung-lab/Agglomeration with MIT License.

- Skeletonization, morphology and functions could be found at https://github.com/seung-lab/RealNeuralNetworks.jl with Apache License 2.0. 
bioRxiv preprint doi: https://doi.org/10.1101/2020.10.28.359620; this version posted February 3, 2021. The copyright holder for this preprint (which was not certified by peer review) is the author/funder, who has granted bioRxiv a license to display the preprint in perpetuity. It is made available under aCC-BY-ND 4.0 International license.

\section{Supplementary Figures}




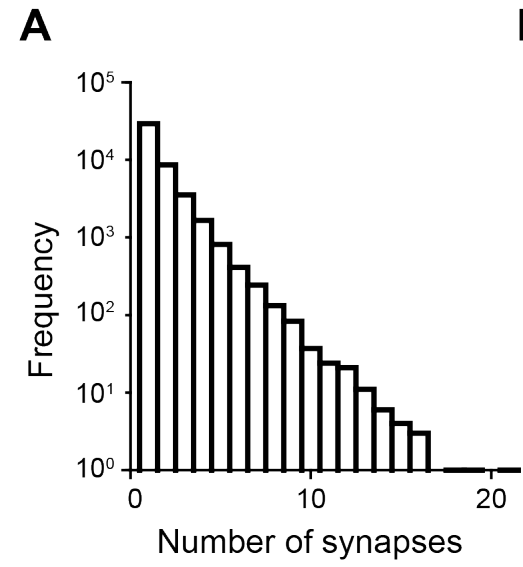

D

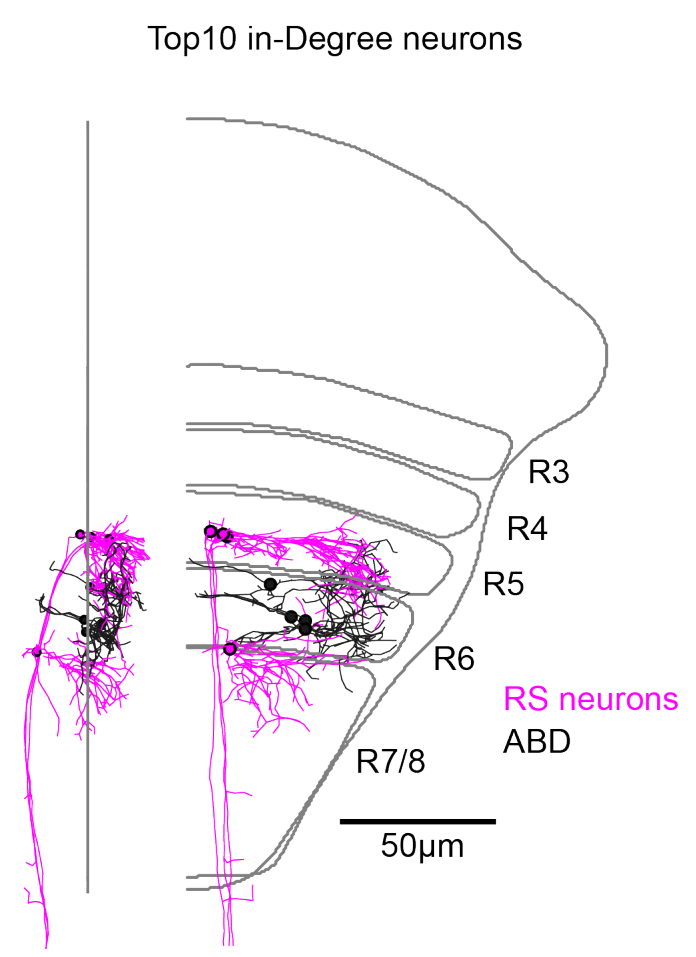

B

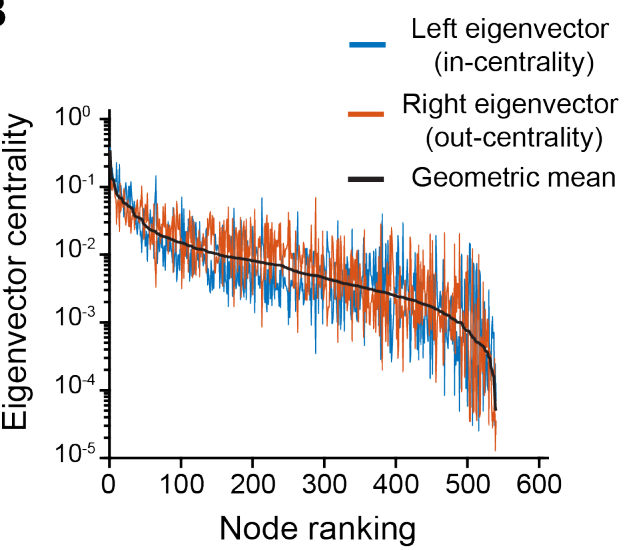

$\mathbf{E}$

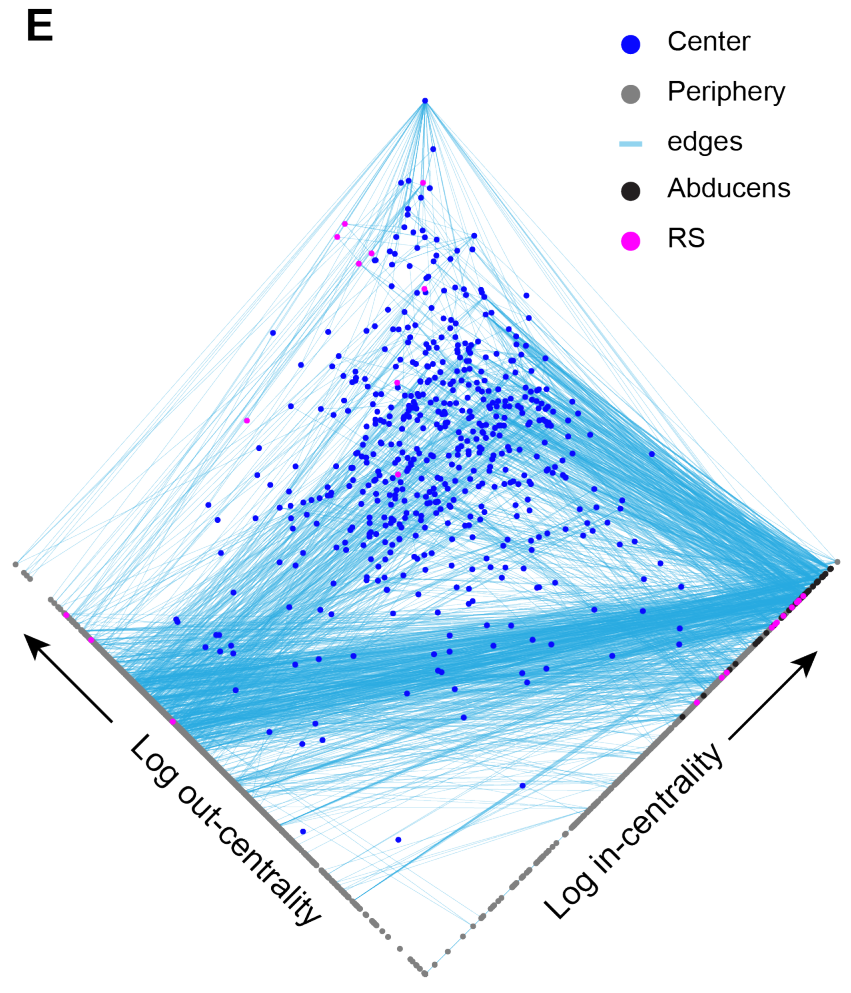

C

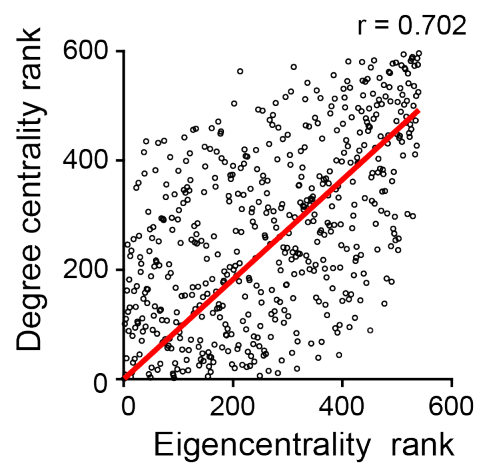

- Center

Periphery

- Abducens

- RS

\section{Figure S1: Important features of the reconstructed network}

A. Frequency distribution of the number of synapses per connection for all neurons in the network with soma in the EM volume.

B. Elements of the left leading eigenvector of the connectivity matrix, the right leading eigenvector, and the geometric mean of these elements (i.e. eigencentrality) for cells ranked by eigencentrality.

C. The rank of neurons when ordered by degree centrality vs eigencentrality.

D. Top10 in-degree neurons 4 are vSPNs and 5 are ABD neurons.

E. Graphical representation of centrality relationships in the network. Dots represent individual neurons and lines represent the connections between neurons. Only edges involving more than 5 synapses shown for clarity. 
A

Light Microscope Larval zebrafish Electron Microscope

(LM) volume reference atlas (EM)volume

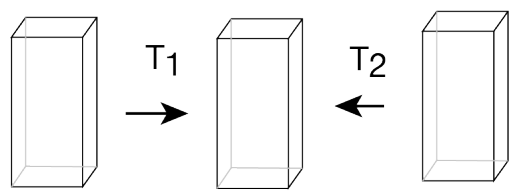

B Functional 2P LM imaging aligned to Z-Brian atlas

Reference atlas Functional imaging

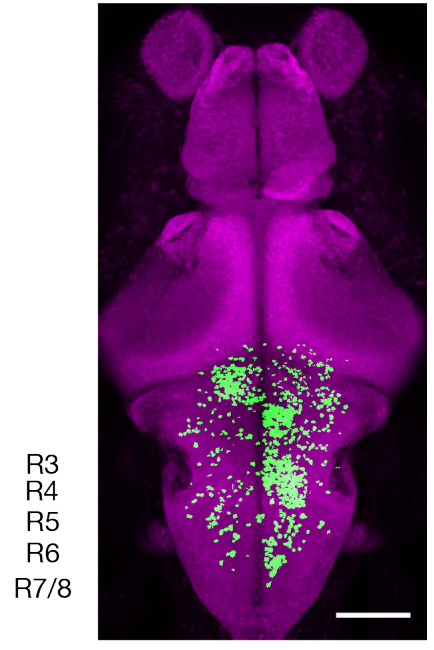

$\mathrm{OB}$

$\mathrm{Te}$

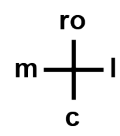

HB

SC

Example eye position neuron

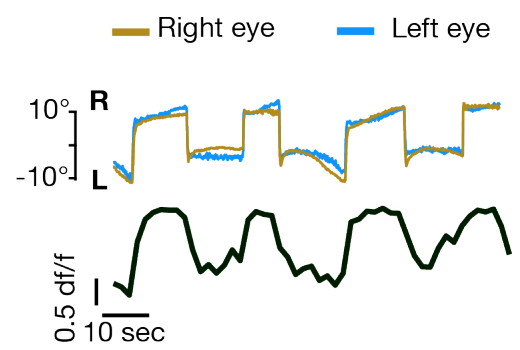

C 3D EM volume registered to Z-Brain atlas

Reference atlas

EM volume
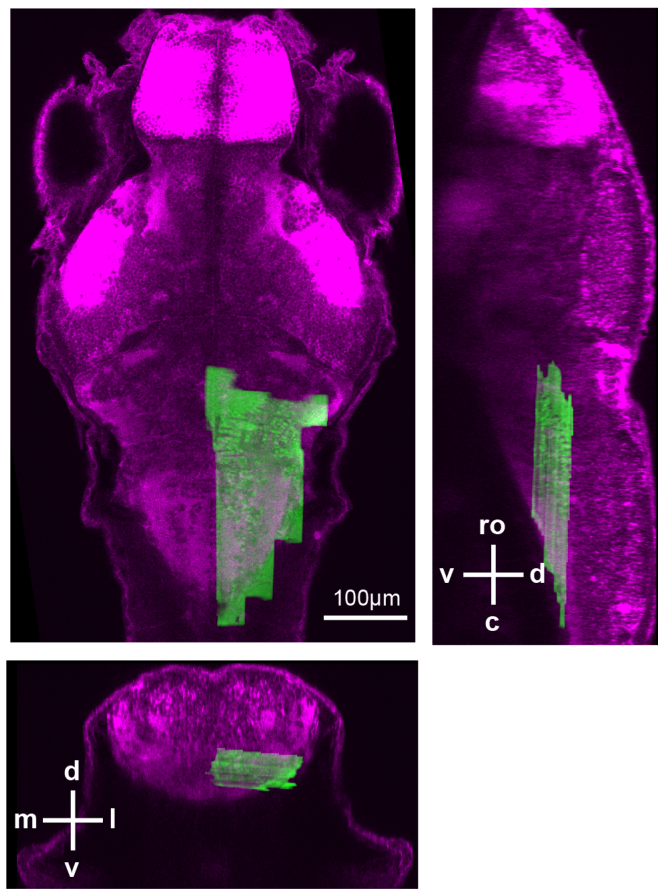

\section{Figure S2: Registration of electron microscopy and light microscopy images to a reference atlas}

A. Schematic indicated separate transformations (T) for light microscopy (LM) images and electron microscopy images onto a common zebrafish brain (Z-Brain) reference atlas.

B. (Top) Maximum intensity projection in the horizontal plane of registered two-photon (2P) functional imaging data (green) onto the Z-Brain reference atlas (magenta). Locations of neurons that were responsive to increase in right eye position are shown. (Bottom) Example activity of a neuron (black) during changes in eye position (blue, yellow). Scale bar $100 \mu \mathrm{m}$.

C. Single plane views (top left: horizontal; top right: sagittal; bottom: coronal) of the registeredEM data set(green) onto Z-Brain reference atlas (magenta). 


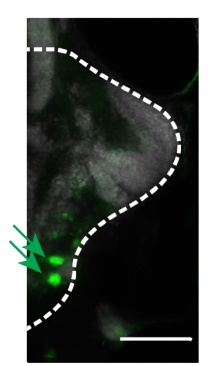

ZBB-m/2-mnx1-GFP

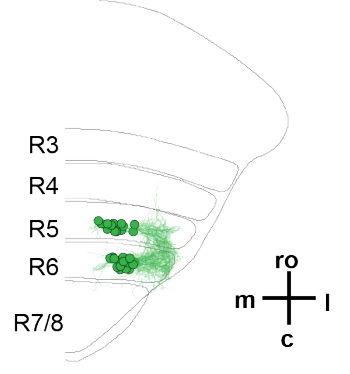

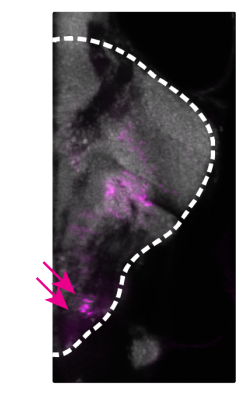

ZBB-evx2-gal4

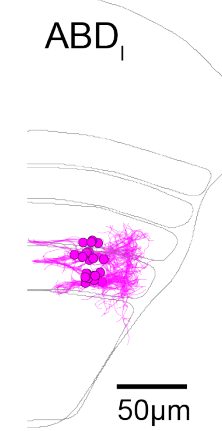

B Descending Octavolateral (DO) neuron identification

Top view $(X Y)$ Saggital view (YZ)
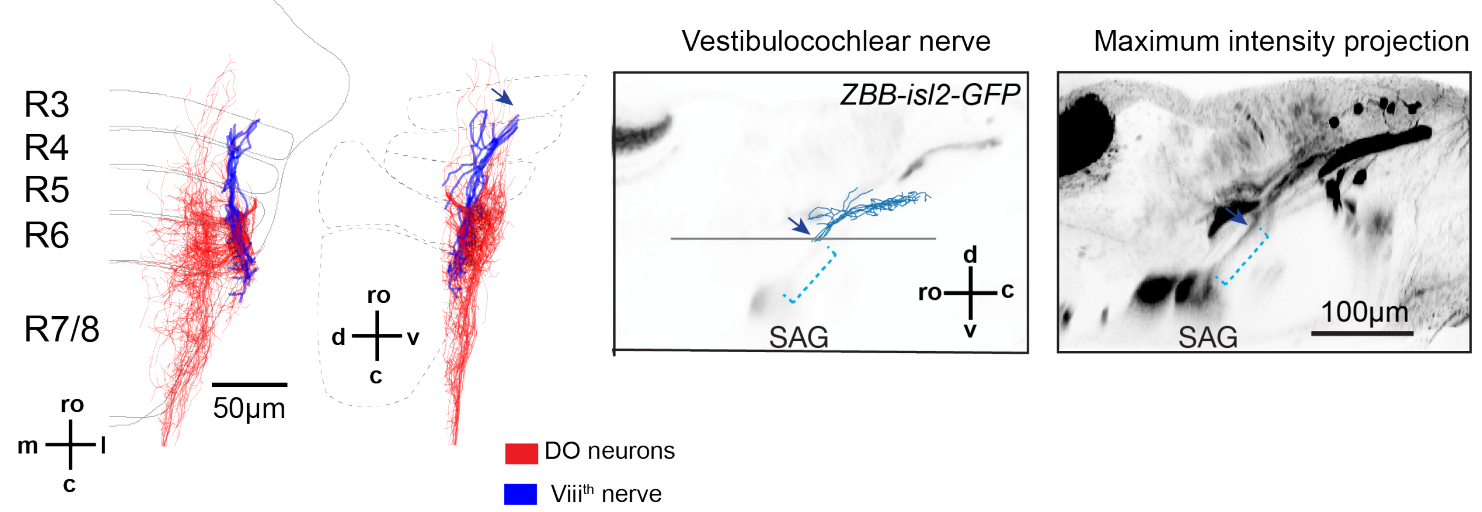

C Zbb-Vglut-DsRed Zbb-gad1b-GFP Zbb-gly2-GFP
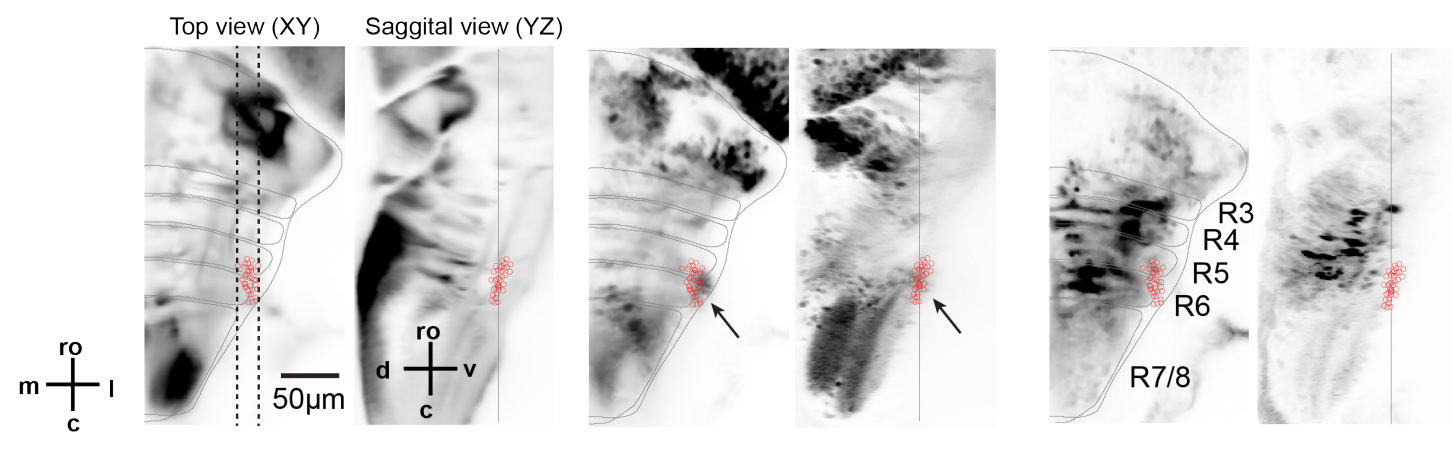

Figure S3: Identification of abducens and descending octaval neurons.

A. Overlap of abducens neurons with transgenic lines registered to the Z-Brain atlas. (Left) Location of abducens motoneurons labelled in the ZBB-ml2-mnx1-GFP transgenic line as indicated in fluorescence micrographs by two clusters of neurons in R5,6 (green arrows). Corresponding locations of ABDM neurons from EM reconstructions are shown in green. (Right) Location of two clusters of abducens internuclear cells labelled in the ZBB-evx2-gal4 transgenic cross (magenta arrows) corresponds with EM reconstructions of ABDI neurons. (ro - rostral; c - causal; m - medial; I - lateral). Scale bar $100 \mu \mathrm{m}$. B. (Left) Reconstruction of descending octaval (DO) neurons (red) along with inputs (blue) from the vestibulocochlear nerve (VIII ${ }^{\text {th }}$ nerve). Side view highlights the ventral exit of the VIII ${ }^{\text {th }}$ nerve in R3 (arrow). Red circles indicate somata, and grey line indicates the mean horizontal plane through the DO soma. (Middle) Single plane (right) maximum intensity projection. SAG - Statoacoustic Ganglia. (ro - rostral; c - causal; $d$ - dorsal; v - ventral). Blue arrow indicates the exit point of the nerve in the reconstructed volume. Blue dotted line indicates the trajectory of the nerve from the SAG.

C. Overlap of DO neuron soma (red circles) with transgenic lines that indicate the location of excitatory (ZBB-Vglut-Dsred) and inhibitory (ZBB-gad1b-GFP, ZBB-gly2-GFP) neurons. The horizontal planes (top view) are the average intensity plane over the rage of DO soma along the dorso-ventral axis. Sagittal view are maximal intensity projections of the planes that contain DO soma (dotted lines). Black arrows indicate the overlap of DO neurons to a GABAergic cluster of neurons. 
bioRxiv preprint doi: https://doi org/10.1101/2020 1028.359620; this version posted February 3, 2021. The copyright holder for this preprint (which was not certified by peer review) is the author/funder, who has granted bioRxiv a license to display the preprint in perpetuity. It is made available under aCC-BY-ND 4.0 International license.

a

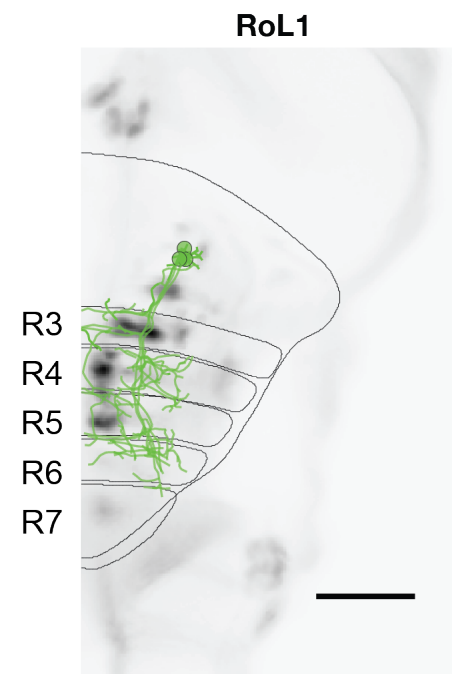

MiV1
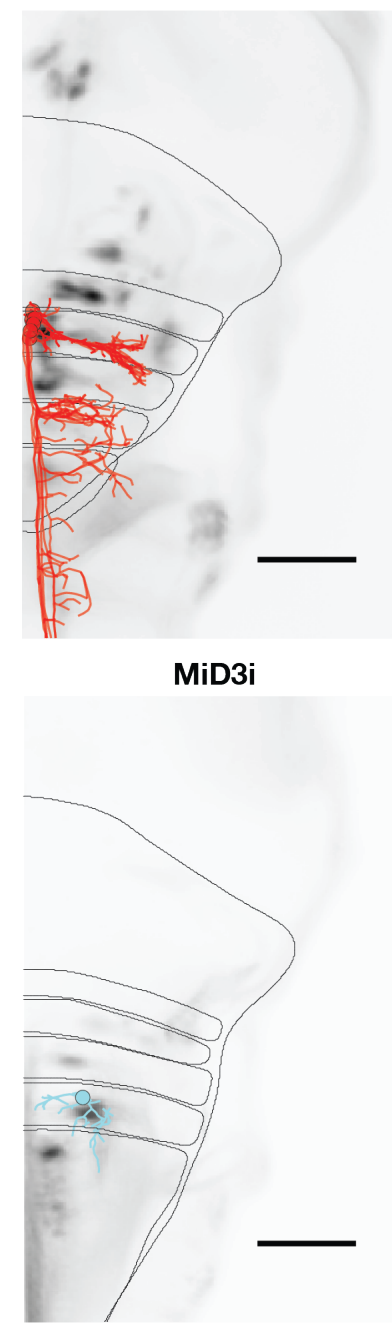

RoV3

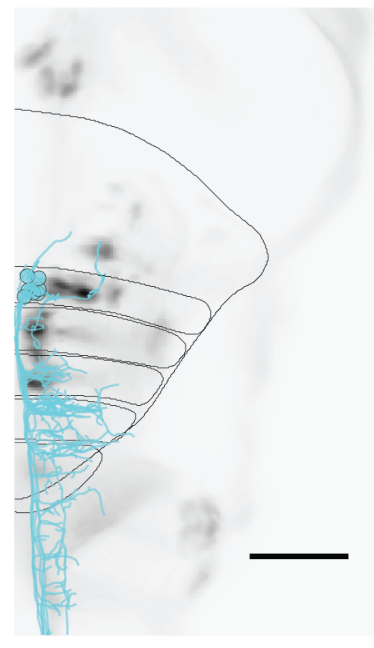

MiV2

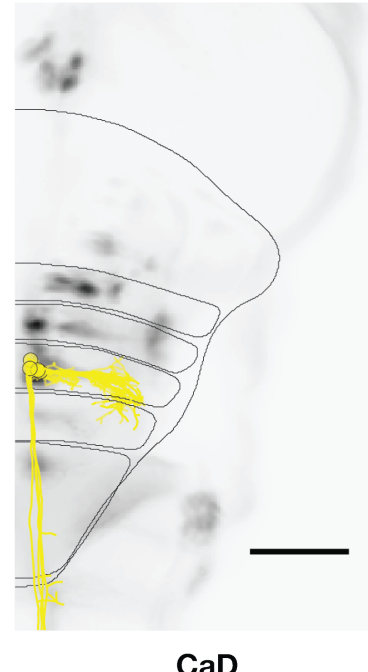

$\mathrm{CaD}$

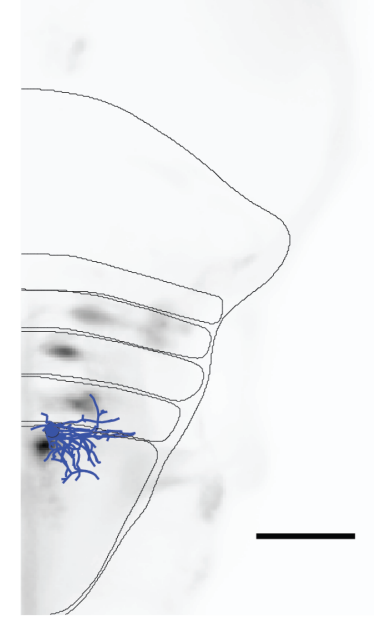

MiM1

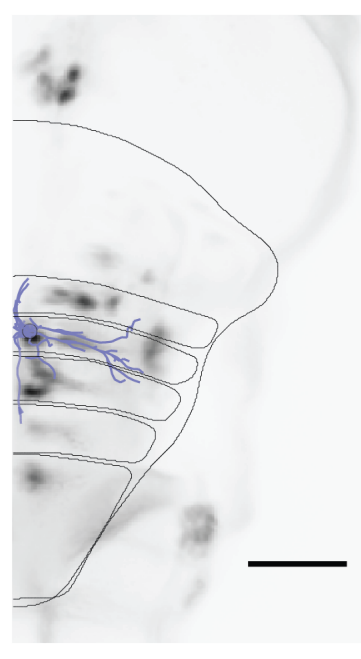

MiD2

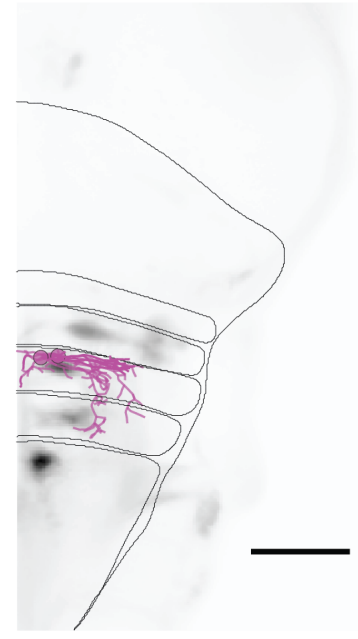

MiT

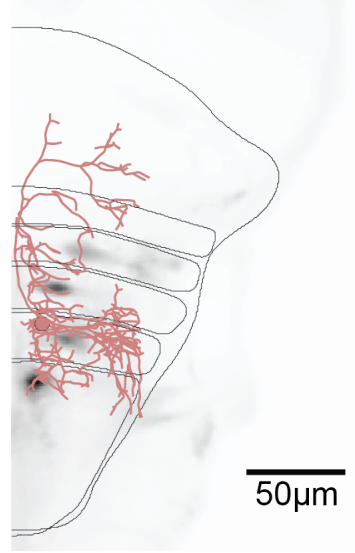

Figure S4: Identification of Reticulospinal (RS) neurons.

A. RS neurons were identified based on their overlap with neurons labeled by spinal backfills that were part of the Z-Brain atlas (ro - rostral; c - causal; m - medial; I - lateral). Scale bar $50 \mu \mathrm{m}$. 
A
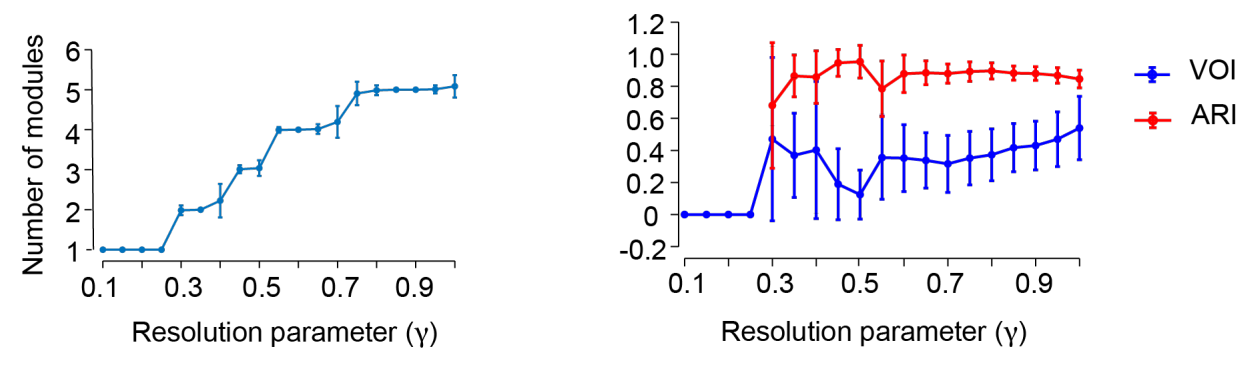

B

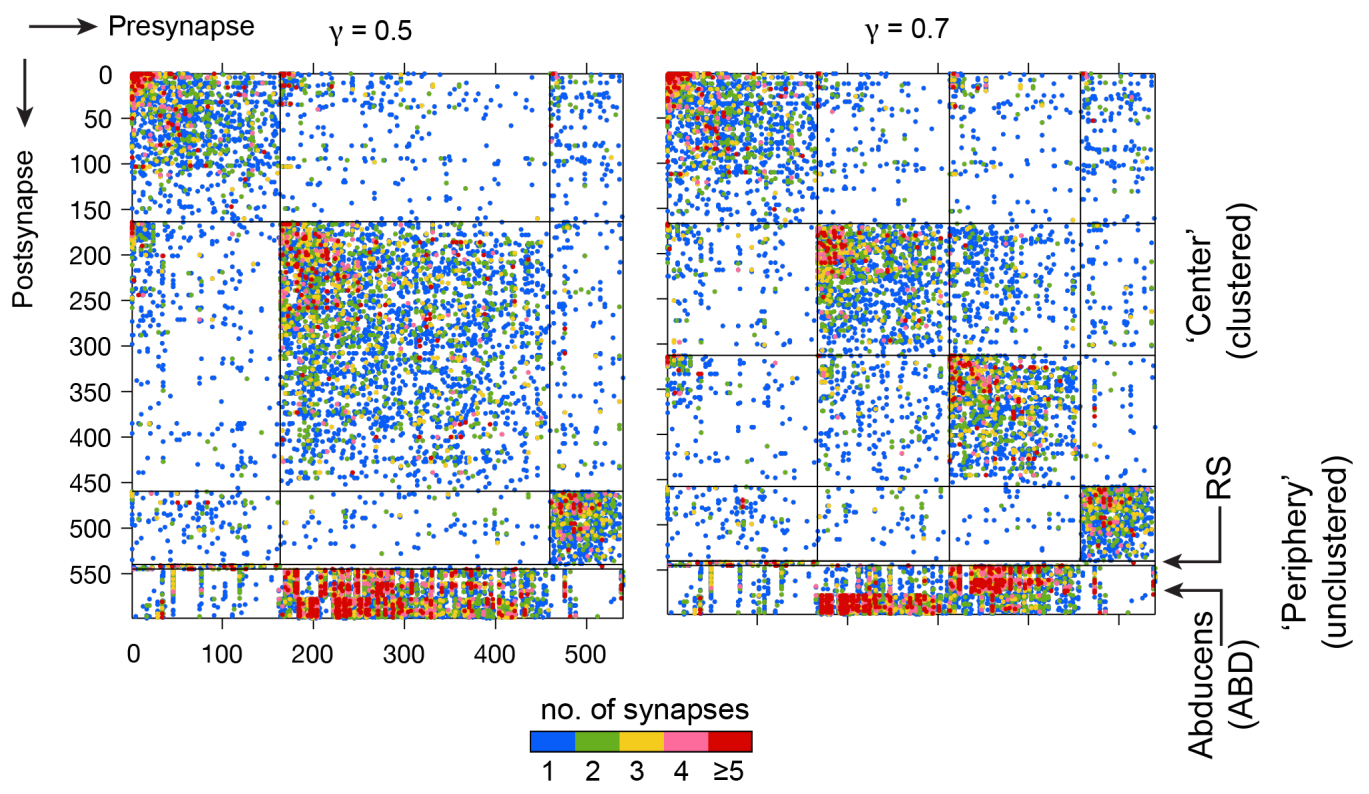

Figure S5: Modular structure at different levels of resolution.

A. Number of modules obtained and robustness of clusters as a function of resolution parameter $\gamma$.

B. Connectivity matrices when clustered for different numbers of modules. At $\gamma=0.7$, emergent modular structure is similar to that as shown when modules in Fig $2(\bmod A, \operatorname{modO})$ and $\mathrm{Fig} 3\left(\operatorname{modO} \mathrm{M}_{\mathrm{M}}, \operatorname{modO}_{\mathrm{I}}\right)$ are clustered in a hierarchical manner. 
a
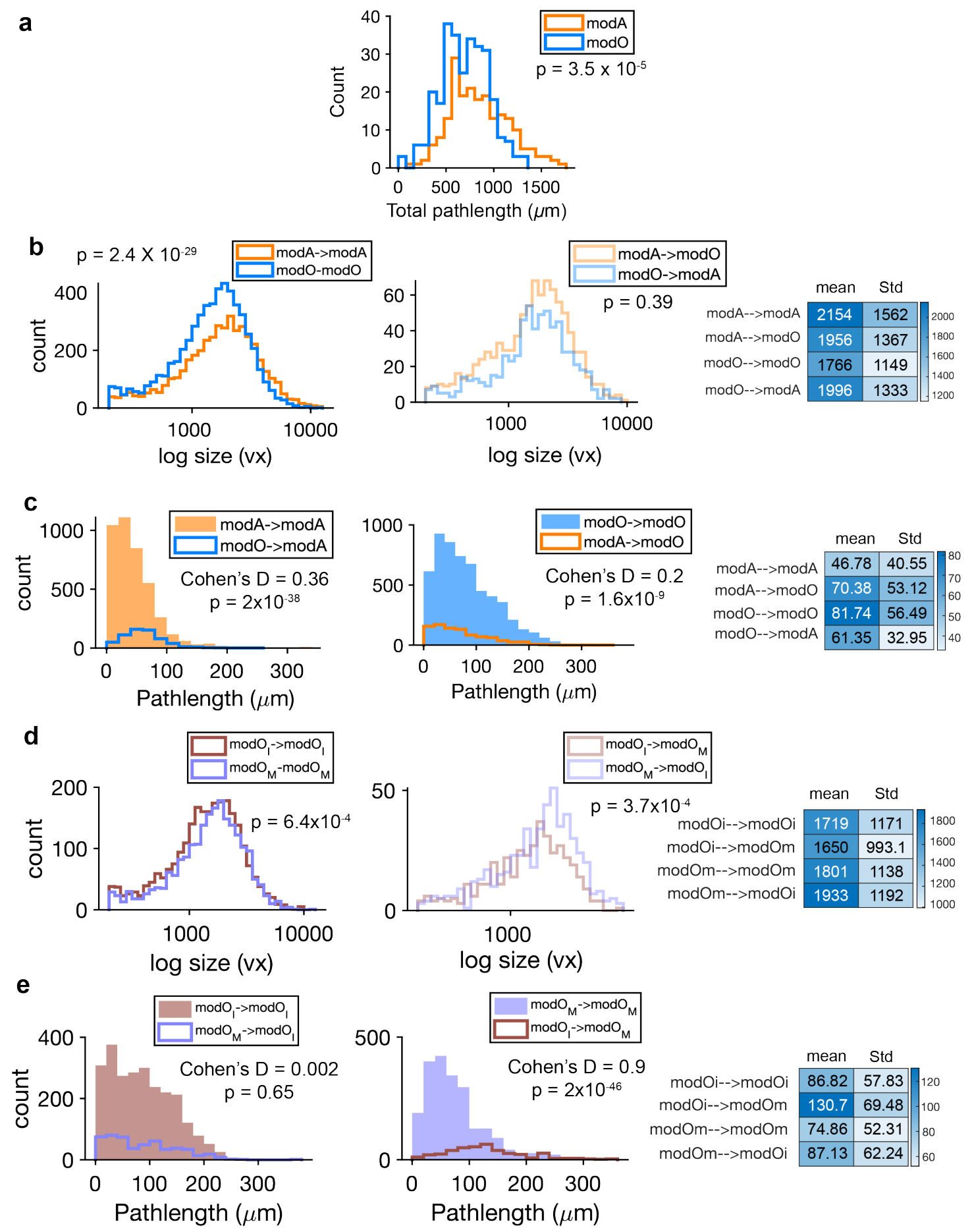

Figure S6: Synapse size and location distribution.

A. Histogram of total pathlength of neurons in $\operatorname{modA}(n=251)$ and $\operatorname{modO}(n=289)$. $P$ is the significance value based on Wilcoxon-rank sum test (RS- test). Cohen's D measures the effect size.

B. Histogram of synaptic size (detected PSD voxels [vx]) for connections within (left) and between (right) modules modA and modO. Table (here and below) summarizes the mean and standard deviations.

C. Histogram of synapses locations i.e. distance from somata to synaptic site along the neurite for neurons in $\bmod A$ and modO.

D. Histogram of synapses size (detected PSD) of neurons within-module (left) and between-modules (right) for modO, and $\operatorname{modO}_{\mathrm{M}}$.

E. Histogram of synapse locations for neurons within the oculomotor module, $\operatorname{modO}_{1}$ and $\operatorname{modO}_{\mathrm{M}}$. 


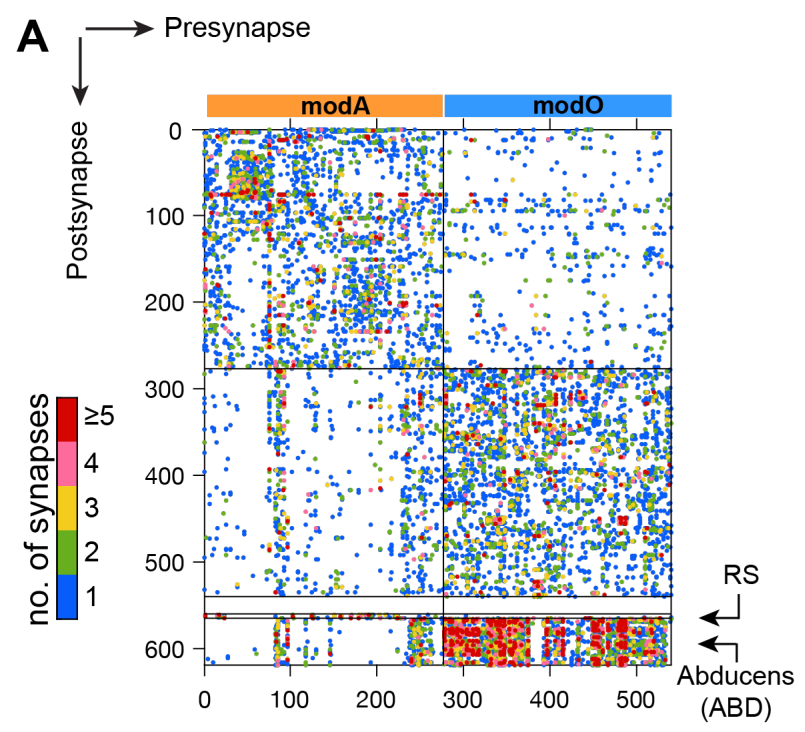

B

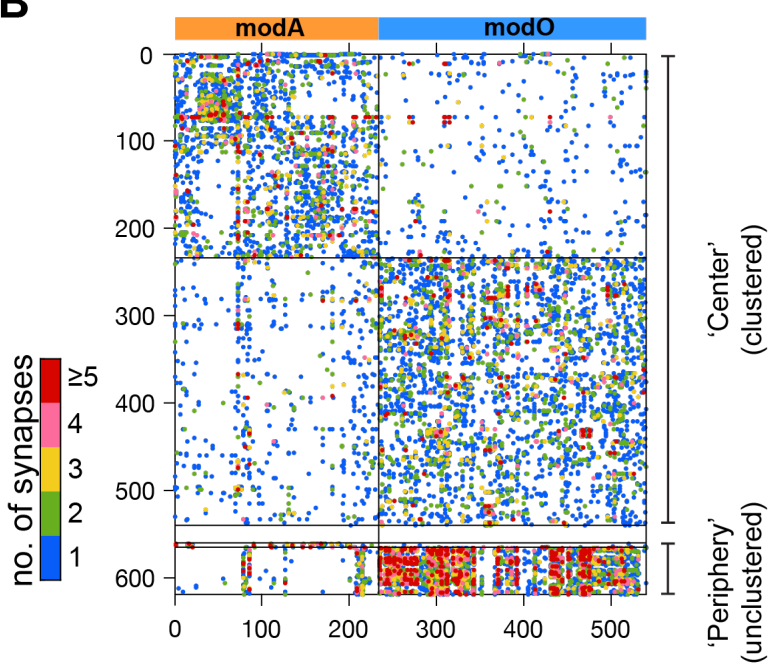

\section{Spectral clustering}

\begin{tabular}{|c|c|c|c|}
\hline \multicolumn{4}{|c|}{ Normalized number of synapses } \\
\hline & $\bmod A$ & $\bmod O$ & \\
\hline $\bmod A$ & 0.06102 & 0.009554 & \\
\hline $\operatorname{modO}$ & 0.01639 & 0.07245 & 0.3 岕 \\
\hline RS & 0.1892 & 0.0327 & $\left|\begin{array}{ll}0.2 & \widehat{D} \\
0.15 & \#\end{array}\right|$ క \\
\hline ABD & 0.04091 & 0.4601 & $\begin{array}{l}0.1 \\
0.05\end{array}$ \\
\hline
\end{tabular}

\section{SBM clustering}

Normalized number of synapses

\begin{tabular}{|c|c|c|c|}
\hline \multirow[b]{2}{*}{$\bmod A$} & $\bmod A$ & $\bmod O$ & \multirow{5}{*}{ 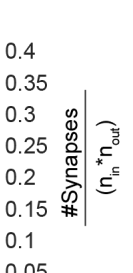 } \\
\hline & 0.07409 & 0.008393 & \\
\hline modo & 0.01131 & 0.06531 & \\
\hline RS & 0.2214 & 0.03007 & \\
\hline & 0.02683 & 0.4119 & \\
\hline
\end{tabular}

Figure S7: Clustering algorithm comparisons.

A. (Left) Connectivity when the center is organized by spectral clustering into two modules. (Right) Normalized number of synapses within and between relevant cell groups.

B. (Left) Connectivity when the center is organized by degree corrected Stochastic Block Matching (SBM) into two modules. (Right) Normalized number of synapses within and between relevant cell groups. 
bioRxiv preprint doi: https://doi.org/10.1101/2020.1028.359620; this version posted February 3, 2021. The copyright holder for this preprint (which was not certified by peer review) is the author/funder, who has granted bioRxiv a license to display the preprint in perpetuity. It is made available under aCC-BY-ND 4.0 International license.

A

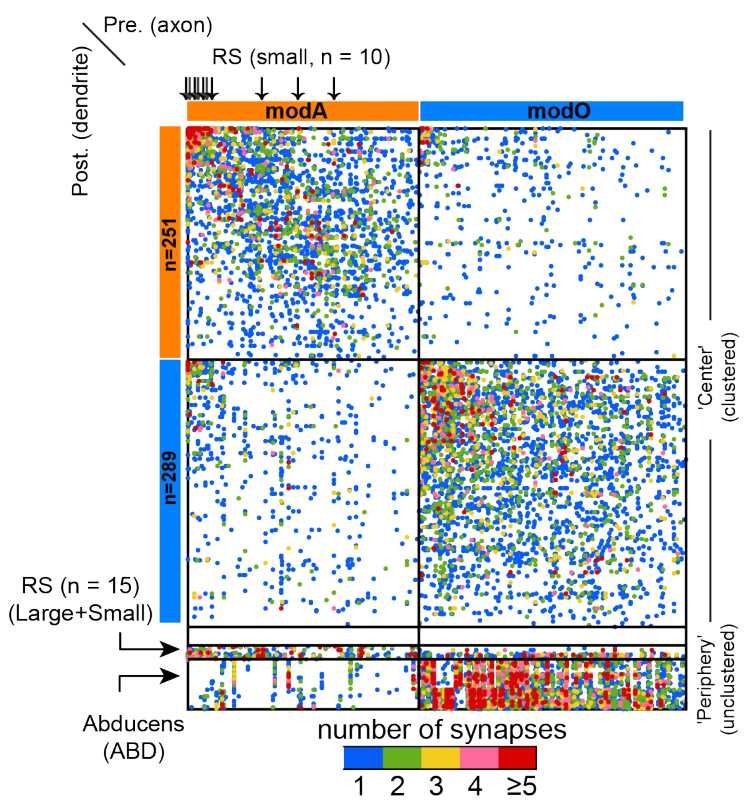

B
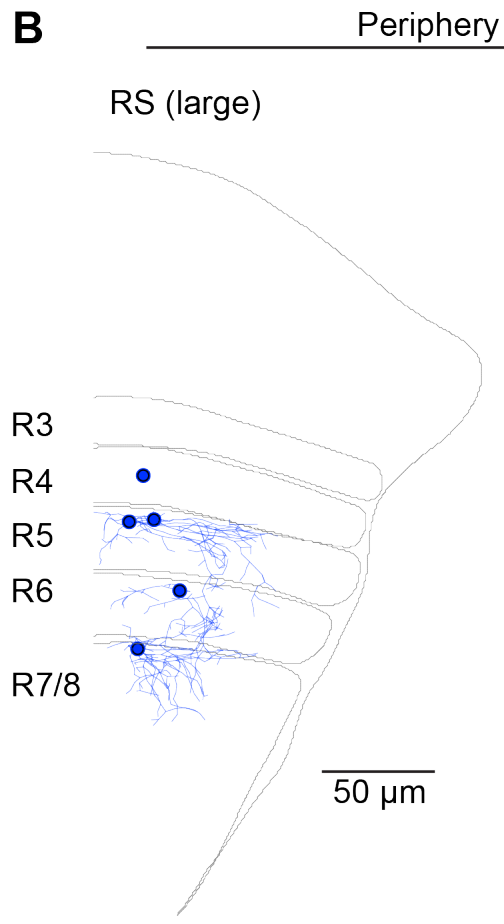

RS (small)

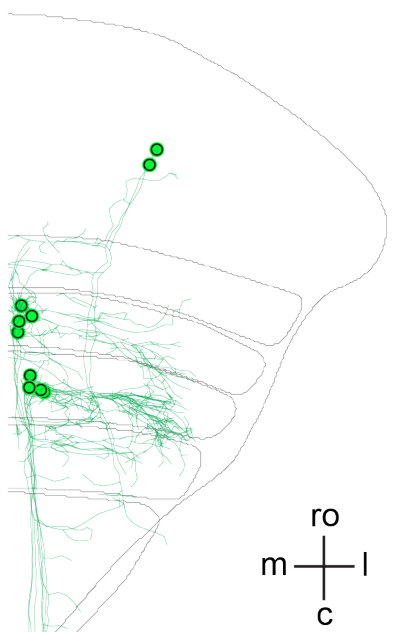

C

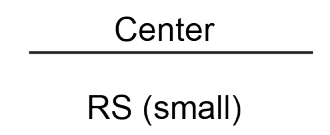

Figure S8: Composition of Axial module $(\bmod A)$.

A. Connectivity matrix (as in Figure. 2) with identification (arrows) of small RS neurons that were part of the center and inclusion into the periphery the remaining small RS neurons along with the large RS neurons.

B. Visualization of the large and small classes of RS neurons in the periphery (ro - rostral; c - causal; $m$ - medial; I - lateral).

C. Visualization of small vSPNs that are part of the 'center' in modA and are indicated by arrows above the rows in (A). 

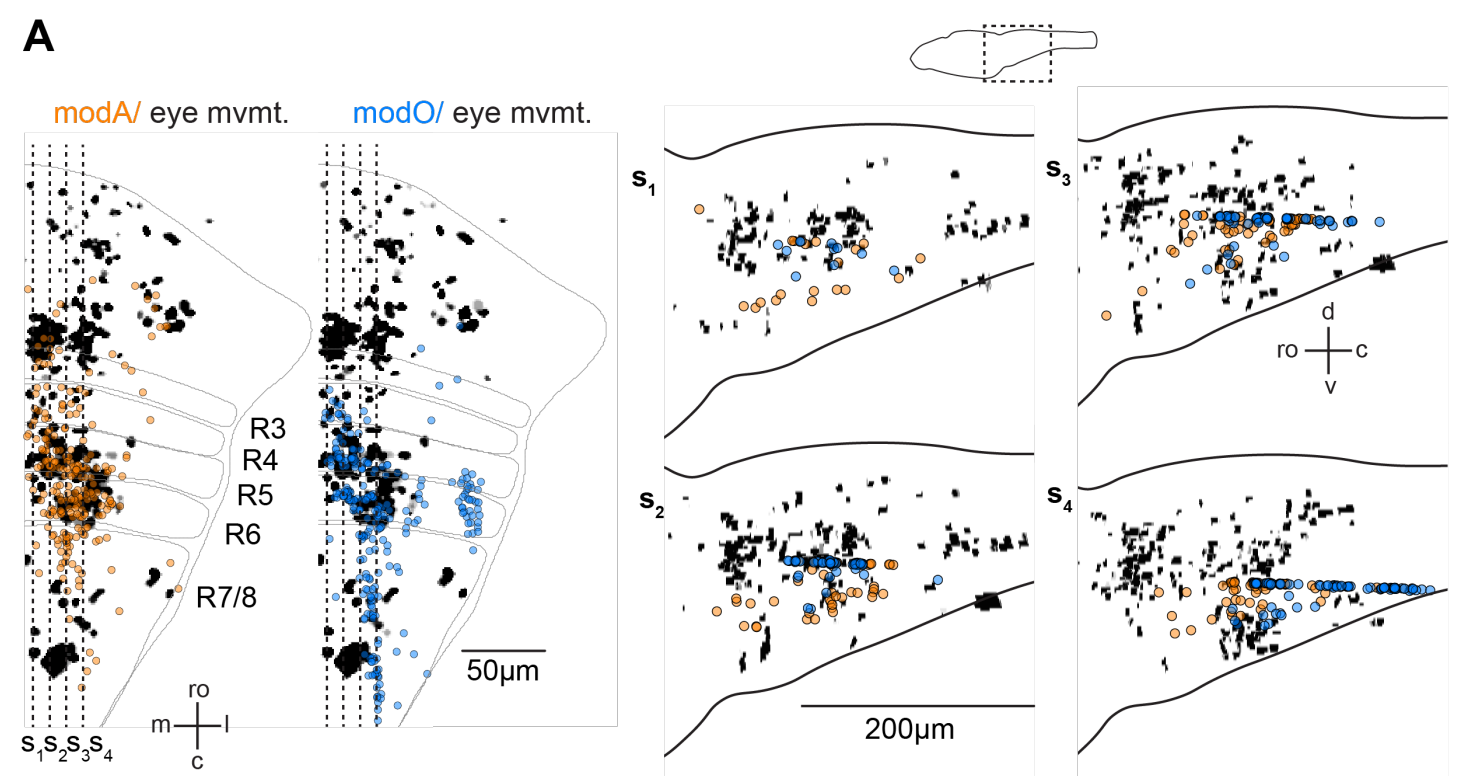

B
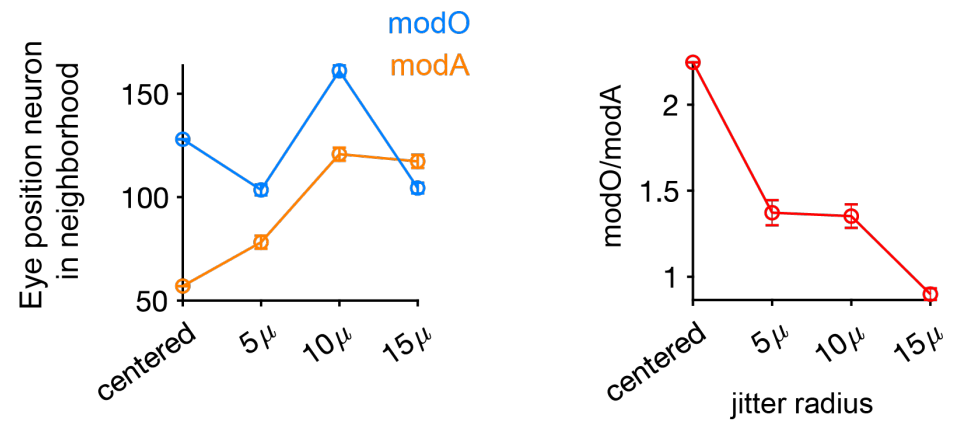

C
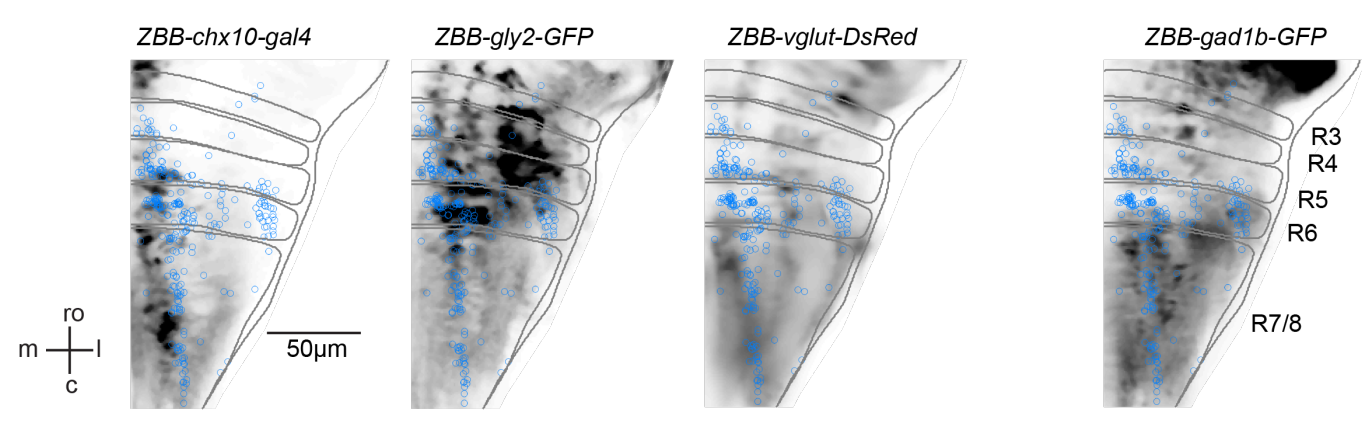

Figure S9: Overlap of modules with functional maps.

A. (Left) Overlap of neurons in modA (orange) and modO (blue) with a maximum intensity projection of neurons with eye movement signals (see Methods), functional map (background). Dotted lines indicate plane at which transverse views are visualized - (Right) Transverse views s1-s4 from left. Soma (foreground) and eye position signals (background) are over a span of $10 \mu \mathrm{m}$ along the medio-lateral axes centered on the dotted lines (ro - rostral; c - causal; m - medial; I - lateral; $\mathrm{d}$ dorsal; v - ventral).

B. Quantification of the overlap of modO neurons from EM to neurons with eye movement signals functional imaging. (Top) Number of eye movement neurons as a function of jitter radius. Error bars are standard deviations of 10 iterations of jitter. (Bottom) ratio of eye position neurons as a function of soma jitter. Overlap was counted using a patch size of $6 \times 6 \times 8 \mu \mathrm{m}$ around each modO neuron.

C. Overlap of modO neurons registered to the Z-Brain atlas to transgenic lines that label neurotransmitter also in the same reference frame. 
A
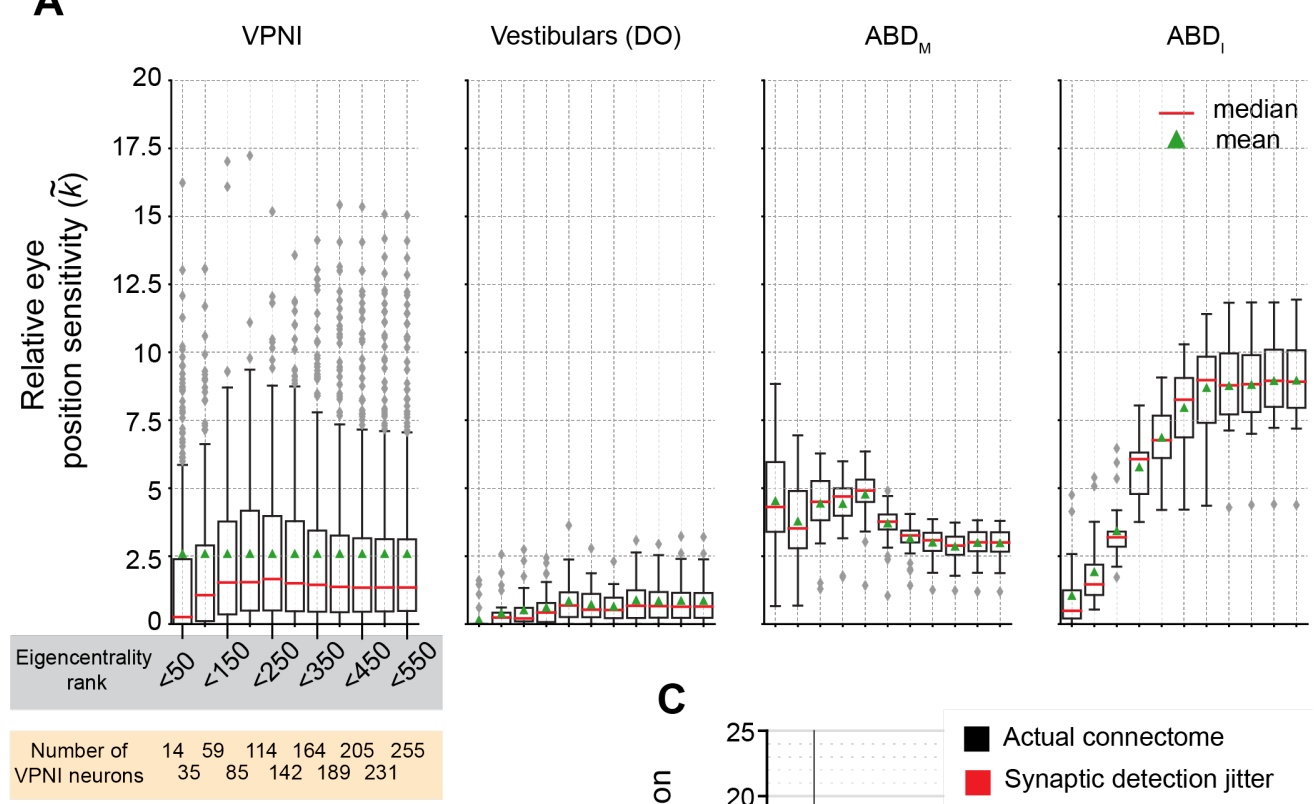

\section{C}

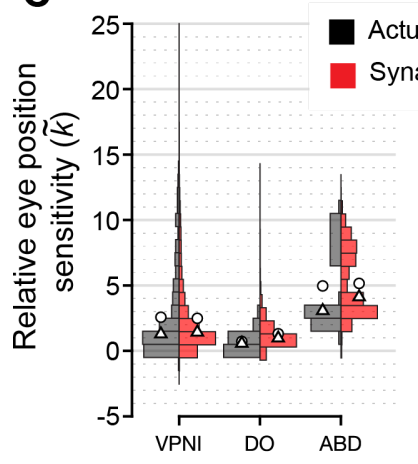

B

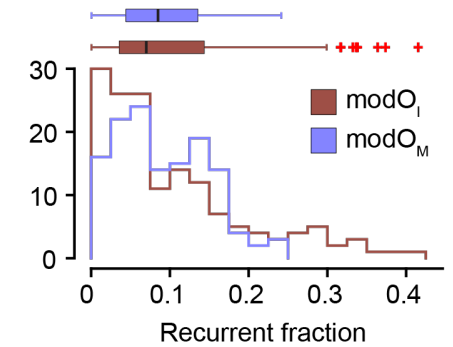

Synaptic detection jitter

D
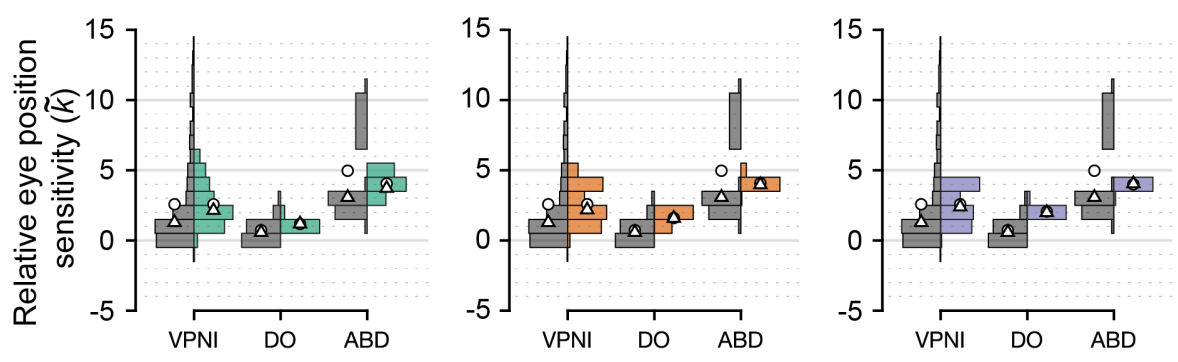

Actual connectome

Potential connectome - $5 \mu \mathrm{m}$

Potential connectome - $2 \mu \mathrm{m}$

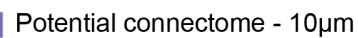

Figure S10: Relative eye position sensitivity distributions for model variants.

A. Box plots of relative eye position sensitivity $(\tilde{k})$ values for all four cell types, VPNIs, DOs, ABDm and ABDi neurons, when different numbers of neurons are used for the center. In these plots, the rightmost box represents the simulation shown in the main paper, when all center neurons are used. Boxes to the left of this indicate simulations run in which only neurons with a more stringent criterion for eigencentrality were included in the center, reducing the number of neurons in the population (number of VPNI model neurons included is shown in the highlighted box). The distributions of relative eye position sensitivity across the four neuronal populations remain consistent even when we shrink the candidate VPNI population by $50 \%$.

B. Histogram of the fraction of all neurons that are recurrent within the identified modules. (left) Recurrent fraction within modO and $\operatorname{modO}_{\mathrm{M}}$. Box plots, above, show medians along with $25^{\text {th }}$ and $75^{\text {th }}$ percentile. Red crosses are the outliers.

C. Distribution of the relative eye position sensitivities $(\tilde{k})$ when the model uses the actual connectivity matrix (black) compared to connectivity matrices generated by simulating small errors in automated synapse detection (red, see Methods). Circles represent the means and triangles represent the medians.

D. Distribution of the relative eye position sensitivities $(\tilde{k})$ when the model uses the actual connectivity matrix (black) compared to connectivity matrices generated considering potential synapses identified with increasingly poor resolution (green, brown, purple). Circles represent medians and triangles represent means. Simulations using connectomes with potential synapses quickly deviate from both those derived from the actual connectome and from the experimental distribution (Figure. 4E) as the potential synapse distance is increased. 


\section{Supplementary Information}

\section{Gamification of the zebrafish dataset and crowdsourced reconstructions}

In addition to in-house reconstructions by experts, zebrafish cells were made available to citizen scientist gamers through the existing retinal crowdsourcing game "Eyewire."

To test out this new dataset only the most experienced players on Eyewire were allowed to participate. A small group of 4 highly experienced players received an invite to test this new dataset. The players were given the title of "Mystic" which was a new status created to enable gameplay, and became the highest achievable rank in the game.

Subsequent Mystic players had to apply for the status to unlock access to the zebrafish dataset within Eyewire. There was a high threshold of achievement required for a player to gain Mystic status. Each player was required to reach the previous highest status within the game, as well as complete $200+$ cubes a month and maintain $95 \%$ accuracy when resolving cell errors.

Once a player was approved by the lab, they were granted access to the zebrafish dataset, and given the option to have a live tutorial session with an Eyewire admin. There was also a pre-recorded tutorial video and written tutorial materials for players who could not attend the live session or who desired a review of the materials. Newly promoted players were also given a special badge, a new chat color, and access to a new chat room exclusive to Mystics. These rewards helped motivate players by showing their elevated status within the game as well as giving them a space to discuss issues specific to the zebrafish dataset.

Cells were parceled out in batches to players. When a cell went live on Eyewire, it could be claimed by any Mystic. Each cell was reconstructed by only one player at a time. Once this player had finished their reconstruction, a second player would check over the cell for errors. After the second check, a final check was done by an Eyewire Admin. To mitigate confusion when claiming cells, a special GUI was built into the game that allowed players to see the status of each cell currently online. A cell could be at one of five statuses - "Need Player A," "Player A," "Need Player B," "Player B," and "Need Admin." These statuses indicated whether a cell needed to be checked, or was in the process of being checked, and whether it needed a first, second, or Admin level check. At each stage the username of the player or Admin who had done a first, second, or final check was also visible. It was made mandatory that the first and second checks were done by two separate players.

Collaboration and feedback were important parts of the checking process. If a player was unsure about an area of the cell they were working on, they could leave a note with a screenshot and detail of the issue, or create an alert that would notify an Admin. If a "Player B" or an Admin noticed a mistake made earlier in the pipeline, they could inform the player of the issue via a "Review" document, or through an in-game notification (player tagging).

To differentiate the zebrafish cells from the regular dataset, each cell was labeled with a Mystic tag. This tag helped to identify the cells as separate from the e2198 retinal Eyewire dataset, and also populated them to a menu of active zebrafish cells within Eyewire.

Players were rewarded for their work in the zebrafish dataset with points. For every edit they made to a cell they received 300 points. Points earned while playing zebrafish were added to a player's overall points score for all gameplay done on Eyewire, and appeared in the Eyewire leaderboard.

The following players in Eyewire were the admins who validated crowd sourced neuronal reconstructions:

Hoodwinked, BenSilverman, Hightower, sarah.morejohn, SeldenK, sorek.m, zorek.m, twisterZ, hjones.jr, devonjones, amy, EinsteintheRapper, zkem, celiad, celiaz,sunreddy, peleaj43, sarahaw

The following players helped reconstruct neurons on Eyewire, collectively called - Mystic players: 
bioRxiv preprint doi: https://doi org/10.1101/2020.10.28.359620; this version posted February 3, 2021. The copyright holder for this preprint

(which was not certified by peer review) is the author/funder, who has granted bioRxiv a license to display the preprint in perpetuity. It is made available under aCC-BY-ND 4.0 International license.

r3, Atani, Nseraf, susi, eldendaf, Frosty, a5hm0r, hiigaran, kinryuu, Manni_Mammut, aesanta1, LotteryDiscountz, galarun, annkri, dragonturtle, LynneC, Cliodhna, jax123, KrzysztofKruk, Kfay, rinda, crazyman4865, JousterL, randompersonjci, Caffeine, Baraka, ggreminder, TR77, hewhoamareismyself, nagilooh, Oppen_heimer, Gruenewitwe, cognaso, twotwos, hawaiisunfun, danielag, lemongrab, zope, MysticM, kondor, frankenmsty, zfishman. 